\title{
SEARCHES FOR ANISOTROPIES IN THE ARRIVAL DIRECTIONS OF THE HIGHEST ENERGY COSMIC RAYS DETECTED BY THE PIERRE AUGER OBSERVATORY
}

A. $\mathrm{Aab}^{42}$, P. Abreu ${ }^{64}$, M. Aglietta ${ }^{53}$, E. J. Ahn ${ }^{81}$, I. Al Samarai ${ }^{29}$, I. F. M. Albuquerque ${ }^{17}$, I. Allekotte ${ }^{1}$, J. Allen ${ }^{84}$, P. Allison ${ }^{86}$, A. Almela ${ }^{11,8}$, J. Alvarez Castillo ${ }^{57}$, J. Alvarez-Muñiz ${ }^{74}$, R. Alves Batista ${ }^{41}$, M. Ambrosio ${ }^{44}$, A. Aminaei ${ }^{58}$, L. Anchordoqui ${ }^{80}$, S. Andringa ${ }^{64}$, C. Aramo ${ }^{44}$, V. M. Aranda ${ }^{71}$, F. Arqueros ${ }^{71}$, H. Asorey ${ }^{1}$, P. Assis ${ }^{64}$, J. Aublin ${ }^{31}$,

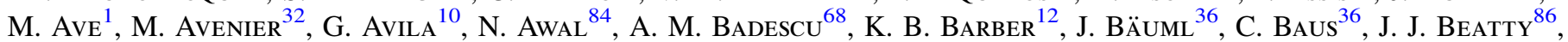
K. H. Becker ${ }^{35}$, J. A. Bellido ${ }^{12}$, C. Berat $^{32}$, M. E. Bertaina ${ }^{53}$, X. Bertou ${ }^{1}$, P. L. Biermann ${ }^{39}$, P. Billoir ${ }^{31}$, S. G. Blaess ${ }^{12}$, M. Blanco ${ }^{31}$, C. Bleve ${ }^{48}$, H. Blümer ${ }^{36,37}$, M. Boháčová ${ }^{27}$, D. BonCIOli ${ }^{52}$, C. Bonifazi ${ }^{23}$, R. Bonino ${ }^{53}$, N. Borodai ${ }^{62}$,

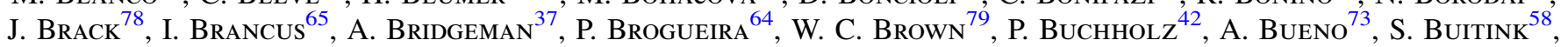
M. Buscemi $^{44}$, K. S. Caballero-Mora ${ }^{55,96}$, B. Caccianiga ${ }^{43}$, L. Caccianiga ${ }^{31}$, M. Candusso ${ }^{45}$, L. Caramete ${ }^{39}$, R. Caruso ${ }^{46}$, A. Castellina ${ }^{53}$, G. Cataldi ${ }^{48}$, L. Cazon ${ }^{64}$, R. Cester $^{47}$, A. G. Chavez ${ }^{56}$, A. Chiavassa ${ }^{53}$, J. A. Chinellato ${ }^{18}$, J. Chudoba ${ }^{27}$, M. Cilmo $^{44}$, R. W. Clay ${ }^{12}$, G. Cocciolo ${ }^{48}$, R. Colalillo ${ }^{44}$, A. Coleman ${ }^{87}$, L. Collica ${ }^{43}$, M. R. ColucCia ${ }^{48}$, R. Conceição ${ }^{64}$, F. Contreras ${ }^{9}$, M. J. Cooper ${ }^{12}$, A. Cordier ${ }^{30}$, S. Coutu ${ }^{87}$, C. E. Covault ${ }^{76}$, J. Cronin ${ }^{88}$, A. Curutiu ${ }^{39}$, R. Dallier ${ }^{34,33}$, B. Daniel ${ }^{18}$, S. Dasso ${ }^{5,3}$, K. Daumiller ${ }^{37}$, B. R. Dawson ${ }^{12}$, R. M. De Almeida ${ }^{24}$, M. De Domenico ${ }^{46}$, S. J. DE Jong ${ }^{58,60}$, J. R. T. De Mello Neto ${ }^{23}$, I. De Mitri ${ }^{48}$, J. De Oliveira ${ }^{24}$, V. De Souza ${ }^{16}$, L. Del Peral ${ }^{72}$, O. DelignY ${ }^{29}$, H. Dembinski ${ }^{37}$, N. Dhital ${ }^{83}$, C. Di Giulio ${ }^{45}$, A. Di Matteo ${ }^{49}$, J. C. Diaz ${ }^{83}$, M. L. Díaz Castro ${ }^{18}$, F. Diogo ${ }^{64}$, C. Dobrigkeit ${ }^{18}$, W. Docters ${ }^{59}$, J. C. D'Olivo ${ }^{57}$, A. Dorofeev ${ }^{78}$, Q. Dorosti Hasankiadeh ${ }^{37}$, M. T. Dova ${ }^{4}$, J. Ebr ${ }^{27}$, R. Engel ${ }^{37}$, M. ERdMann ${ }^{40}$, M. Erfani ${ }^{42}$, C. O. Escobar ${ }^{81,18}$, J. Espadanal ${ }^{64}$, A. Etchegoyen ${ }^{8,11}$, P. FACAL SAN Luis ${ }^{88}$, H. FAlcke ${ }^{58,61,60}$, K. FAnG ${ }^{88}$, G. FARRAR ${ }^{84}$, A. C. FAuth ${ }^{18}$, N. FAzzini ${ }^{81}$, A. P. Ferguson ${ }^{76}$, M. Fernandes $^{23}$, B. FicK ${ }^{83}$, J. M. Figueira ${ }^{8}$, A. Filevich ${ }^{8}$, A. Filipčič ${ }^{69,70}$, B. D. Fox ${ }^{89}$, O. Fratu ${ }^{68}$, M. M. Freire ${ }^{6}$, U. Fröhlich ${ }^{42}$, B. Fuchs ${ }^{36}$, T. Fujil ${ }^{88}$, R. Gaior ${ }^{31}$, B. García ${ }^{7}$, D. Garcia- Gamez ${ }^{30}$, D. Garcia- Pinto ${ }^{71}$, G. Garilli ${ }^{46}$, A. Gascon Bravo ${ }^{73}$, F. Gate ${ }^{34}$, H. Gemmeke ${ }^{38}$, P. L. Ghia ${ }^{31}$, U. Giaccari ${ }^{23}$, M. Giammarchi ${ }^{43}$, M. Giller ${ }^{63}$, C. Glaser ${ }^{40}$, H. Glass ${ }^{81}$, M. Gómez Berisso ${ }^{1}$, P. F. Gómez Vitale ${ }^{10}$, P. Gonçalves ${ }^{64}$, J. G. Gonzalez ${ }^{36}$, N. González ${ }^{8}$, B. Gookin ${ }^{78}$, J. Gordon ${ }^{86}$, A. Gorgi ${ }^{53}$, P. Gorham ${ }^{89}$, P. GoufFON ${ }^{17}, \mathrm{~S}_{\text {G Grebe }}{ }^{58,60}$, N. Griffith ${ }^{86}$, A. F. Grillo ${ }^{52}$, T. D. Grubb ${ }^{12}$, F. Guarino ${ }^{44}$, G. P. Guedes ${ }^{19}$, M. R. Hampel ${ }^{8}$, P. Hansen ${ }^{4}$, D. Harari ${ }^{1}$, T. A. Harrison ${ }^{12}$, S. Hartmann ${ }^{40}$, J. L. Harton ${ }^{78}$, A. Haungs ${ }^{37}$, T. Hebbeker ${ }^{40}$, D. Heck ${ }^{37}$, P. Heimann ${ }^{42}$, A. E. Herve ${ }^{37}$,

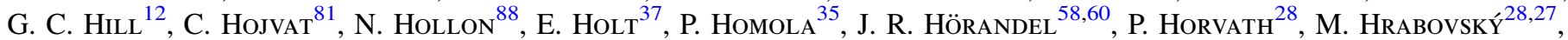

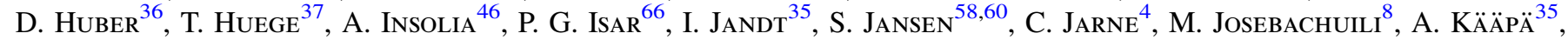
O. Kambeitz ${ }^{36}$, K. H. Kampert ${ }^{35}$, P. Kasper ${ }^{81}$, I. Katkov ${ }^{36}$, B. Kégl ${ }^{30}$, B. Keilhauer ${ }^{37}$, A. Keivani ${ }^{87}$, E. Kemp ${ }^{18}$, R. M. Kieckhafer ${ }^{83}$, H. O. Klages ${ }^{37}$, M. Kleifges ${ }^{38}$, J. Kleinfeller ${ }^{9}$, R. Krause ${ }^{40}$, N. Krohm ${ }^{35}$, O. Krömer ${ }^{38}$, D. Kruppke- Hansen ${ }^{35}$, D. Kuempel ${ }^{40}$, N. Kunka ${ }^{38}$, D. LaHurd ${ }^{76}$, L. Latronico ${ }^{53}$, R. Lauer ${ }^{91}$, M. Lauscher ${ }^{40}$, P. Lautridou ${ }^{34}$, S. Le $\mathrm{COZ}^{32}$, M. S. A. B. LeÃo ${ }^{14}$, D. Lebrun ${ }^{32}$, P. Lebrun ${ }^{81}$, M. A. Leigui De Oliveira ${ }^{22}$,

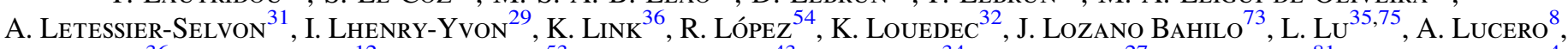

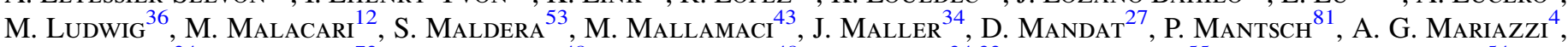
V. Marin ${ }^{34}$, I. C. Mariş ${ }^{73}$, G. Marsella ${ }^{48}$, D. Martello ${ }^{48}$, L. Martin ${ }^{34,33}$, H. Martinez ${ }^{55}$, O. Martínez Bravo ${ }^{54}$,

D. Martraire ${ }^{29}$, J. J. Masías Meza ${ }^{3}$, H. J. Mathes ${ }^{37}$, S. Mathys ${ }^{35}$, J. Matthews ${ }^{82}$, J. A. J. Matthews ${ }^{91}$, G. Matthiae ${ }^{45}$, D. Maurel $^{36}$, D. Maurizio ${ }^{13}$, E. Mayotte ${ }^{77}$, P. O. Mazur ${ }^{81}$, C. Medina ${ }^{77}$, G. Medina- Tanco ${ }^{57}$, R. Meissner ${ }^{40}$, M. Melissas $^{36}$, D. Melo ${ }^{8}$, A. Menshikov ${ }^{38}$, S. Messina ${ }^{59}$, R. Meyhandan ${ }^{89}$, S. Mićanović ${ }^{25}$, M. I. Micheletti ${ }^{6}$, L. Middendorf $^{40}$, I. A. Minaya ${ }^{71}$, L. Miramonti ${ }^{43}$, B. Mitrica ${ }^{65}$, L. Molina- Bueno ${ }^{73}$, S. Mollerach $^{1}$, M. Monasor ${ }^{88}$, D. Monnier Ragaigne ${ }^{30}$, F. Montanet ${ }^{32}$, C. Morello $^{53}$, M. Mostafá ${ }^{87}$, C. A. Moura ${ }^{22}$, M. A. Muller ${ }^{18,21}$, G. Müller ${ }^{40}$, S. Müller ${ }^{37}$, M. MünChmeYer ${ }^{31}$, R. Mussa ${ }^{47}$, G. Navarra ${ }^{53,92}$, S. Navas ${ }^{73}$, P. Necesal ${ }^{27}$ L. Nellen $^{57}$, A. Nelles ${ }^{58,60}$, J. Neuser ${ }^{35}$, P. H. NGuYen ${ }^{12}$, M. NiechCiol ${ }^{42}$, L. Niemietz ${ }^{35}$, T. NigGemann ${ }^{40}$, D. Nitz ${ }^{83}$, D. NoseK ${ }^{26}$, V. Novotny ${ }^{26}$, L. NožKa $^{28}$, L. Ochilo ${ }^{42}$, F. Oikonomou ${ }^{87}$, A. Olinto ${ }^{88}$, M. Oliveira ${ }^{64}$, N. Pacheco ${ }^{72}$, D. PaKK Selmi- Dei ${ }^{18}$, M. Palatka ${ }^{27}$,

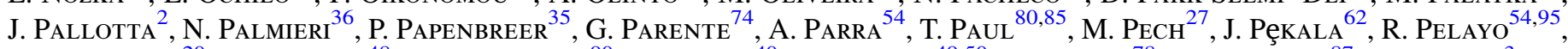
I. M. Pepe ${ }^{20}$, L. Perrone ${ }^{48}$, E. Petermann ${ }^{90}$, C. Peters ${ }^{40}$, S. Petrera ${ }^{49,50}$, Y. Petrov ${ }^{78}$, J. Phuntsok ${ }^{87}$, R. Piegaia ${ }^{3}$,

T. Pierog ${ }^{37}$, P. Pieroni ${ }^{3}$, M. Pimenta ${ }^{64}$, V. Pirronello ${ }^{46}$, M. Platino ${ }^{8}$, M. Plum ${ }^{40}$, A. Porcelli ${ }^{37}$, C. Porowski ${ }^{62}$,

R. R. Prado ${ }^{16}$, P. Privitera ${ }^{88}$, M. Prouza ${ }^{27}$, V. Purrello ${ }^{1}$, E. J. Quel ${ }^{2}$, S. Querchfeld ${ }^{35}$, S. Quinn ${ }^{76}$, J. Rautenberg ${ }^{35}$, O. Ravel ${ }^{34}$, D. Ravignani ${ }^{8}$, B. ReVEnu ${ }^{34}$, J. RidKy ${ }^{27}$, S. Riggi ${ }^{46}$, M. Risse ${ }^{42}$, P. Ristori ${ }^{2}$, V. Rizi ${ }^{49}$,

W. Rodrigues de Carvalho ${ }^{74}$, G. Rodriguez Fernandez ${ }^{45}$, J. Rodriguez Rojo ${ }^{9}$, M. D. Rodríguez- Frías ${ }^{72}$, D. Rogozin ${ }^{37}$, G. $\operatorname{Ros}^{72}$, J. Rosado ${ }^{71}$, T. Rossler ${ }^{28}$, M. Roth ${ }^{37}$, E. Roulet ${ }^{1}$, A. C. Rovero ${ }^{5}$, S. J. SAFFi ${ }^{12}$, A. SAFtoiu ${ }^{65}$, F. SAlamida ${ }^{29}$, H. Salazar ${ }^{54}$, A. Saleh ${ }^{70}$, F. Salesa Greus ${ }^{87}$, G. Salina ${ }^{45}$, F. Sánchez ${ }^{8}$, P. Sanchez- Lucas ${ }^{73}$, C. E. Santo ${ }^{64}$, E. Santos ${ }^{18}$,

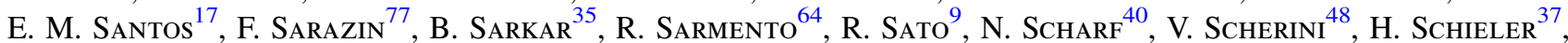

P. Schiffer ${ }^{41}$, D. Schmidt ${ }^{37}$, O. Scholten ${ }^{59,97}$, H. Schoorlemmer ${ }^{89,58,60}$, P. Schovánek ${ }^{27}$, F. G. SchröDer ${ }^{37}$, A. Schulz ${ }^{37}$, J. Schulz ${ }^{58}$, J. Schumacher ${ }^{40}$, S. J. Sciutto ${ }^{4}$, A. Segreto ${ }^{51}$, M. Settimo ${ }^{31}$, A. Shadkam ${ }^{82}$, R. C. Shellard ${ }^{13}$, I. Sidelnik ${ }^{1}$, G. Sigl ${ }^{41}$, O. Sima ${ }^{67}$, A. ŚmiaŁKowski ${ }^{63}$, R. ŠmídA ${ }^{37}$, G. R. Snow ${ }^{90}$, P. Sommers ${ }^{87}$, J. Sorokin ${ }^{12}$, R. Squartini ${ }^{9}$,

Y. N. Srivastava ${ }^{85}$, S. Stanič ${ }^{70}$, J. Stapleton ${ }^{86}$, J. Stasielak ${ }^{62}$, M. Stephan ${ }^{40}$, A. Stutz ${ }^{32}$, F. Suarez ${ }^{8}$, T. SuOmiü̈rvi ${ }^{29}$, 
A. D. Supanitsky ${ }^{5}$, M. S. Sutherland ${ }^{86}$, J. Swain $^{85}$, Z. Szadkowski ${ }^{63}$, M. Szuba ${ }^{37}$, O. A. Taborda ${ }^{1}$, A. Tapia ${ }^{8}$, A. Tepe ${ }^{42}$, V. M. Theodoro ${ }^{18}$, C. Timmermans ${ }^{60,58}$, C. J. Todero Peixoto ${ }^{15}$, G. Toma ${ }^{65}$, L. Tomankova ${ }^{37}$, B. Tomé ${ }^{64}$, A. Tonachini ${ }^{47}$, G. Torralba Elipe ${ }^{74}$, D. Torres Machado ${ }^{23}$, P. Travnicek $^{27}$, E. Trovato $^{46}$, R. Ulrich ${ }^{37}$, M. UnGer $^{37,84}$, M. Urban $^{40}$, J. F. VAldés Galicia ${ }^{57}$, I. VAliño ${ }^{74}$, L. VAlore ${ }^{44}$, G. VAN AaR ${ }^{58}$, P. van Bodegom ${ }^{12}$, A. M. VAN DEN Berg ${ }^{59}$, S. VAN Velzen ${ }^{58}$, A. VAN Vliet ${ }^{41}$, E. Varela ${ }^{54}$, B. Vargas Cárdenas ${ }^{57}$, G. VArner ${ }^{89}$, J. R. Vázquez ${ }^{71}$, R. A. VázQuez ${ }^{74}$, D. Veberič ${ }^{30}$, V. VERZI ${ }^{45}$, J. VICHA $^{27}$, M. VIDELA $^{8}$, L. VILlASE NOR $^{56}$, B. VLCEK $^{72}$, S. VOROBIOV $^{70}$, H. WAHLBERG $^{4}$, O. WAINBERG $^{8,11}$, D. WALZ $^{40}$,

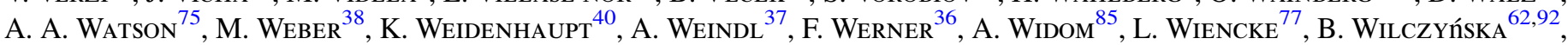
H. Wilczyński ${ }^{62}$, C. Williams ${ }^{88}$, T. Winchen ${ }^{35}$, D. WittKowski ${ }^{35}$, B. Wundheiler ${ }^{8}$, S. WyKes ${ }^{58}$, T. YAMAMOTO $^{88,93}$, T. YAPICI ${ }^{83}$, G. YuAN ${ }^{82}$, A. YushKov ${ }^{42}$, B. Zamorano ${ }^{73}$, E. Zas ${ }^{74}$, D. ZAVRTANiK ${ }^{70,69}$, M. ZaVRTANIK ${ }^{69,70}$, A. ZEPEDA ${ }^{55,94}$,

$$
\text { J. Zhou }{ }^{88} \text {, Y. Zhu }{ }^{38} \text {, M. Zimbres Silva }{ }^{18} \text {, M. Ziolkowski }{ }^{42} \text {, F. Zuccarello }{ }^{46}
$$

(The Pierre Auger Collaboration)

${ }^{1}$ Centro Atómico Bariloche and Instituto Balseiro (CNEA-UNCuyo-CONICET), San Carlos de Bariloche, Argentina

${ }^{2}$ Centro de Investigaciones en Láseres y Aplicaciones, CITEDEF and CONICET, Argentina

${ }^{3}$ Departamento de Física, FCEyN, Universidad de Buenos Aires and CONICET, Argentina

${ }^{4}$ IFLP, Universidad Nacional de La Plata and CONICET, La Plata, Argentina

${ }^{5}$ Instituto de Astronomía y Física del Espacio (IAFE, CONICET-UBA), Buenos Aires, Argentina

${ }^{6}$ Instituto de Física de Rosario (IFIR) - CONICET/U.N.R. and Facultad de Ciencias Bioquímicas y Farmacéuticas U.N.R., Rosario, Argentina

${ }^{7}$ Instituto de Tecnologías en Detección y Astropartículas (CNEA, CONICET, UNSAM), and National Technological University, Faculty Mendoza (CONICET/ CNEA), Mendoza, Argentina

${ }^{8}$ Instituto de Tecnologías en Detección y Astropartículas (CNEA, CONICET, UNSAM), Buenos Aires, Argentina

${ }^{9}$ Observatorio Pierre Auger, Malargüe, Argentina

${ }^{10}$ Observatorio Pierre Auger and Comisión Nacional de Energía Atómica, Malargüe, Argentina

${ }^{11}$ Universidad Tecnológica Nacional-Facultad Regional Buenos Aires, Buenos Aires, Argentina ${ }^{12}$ University of Adelaide, Adelaide, S.A., Australia

${ }^{13}$ Centro Brasileiro de Pesquisas Fisicas, Rio de Janeiro, RJ, Brazil

${ }^{14}$ Faculdade Independente do Nordeste, Vitória da Conquista, Brazil

${ }^{15}$ Universidade de São Paulo, Escola de Engenharia de Lorena, Lorena, SP, Brazil

${ }^{16}$ Universidade de São Paulo, Instituto de Física de São Carlos, São Carlos, SP, Brazil

${ }^{17}$ Universidade de São Paulo, Instituto de Física, São Paulo, SP, Brazil

${ }^{18}$ Universidade Estadual de Campinas, IFGW, Campinas, SP, Brazil

${ }^{19}$ Universidade Estadual de Feira de Santana, Brazil

${ }^{20}$ Universidade Federal da Bahia, Salvador, BA, Brazil

${ }^{21}$ Universidade Federal de Pelotas, Pelotas, RS, Brazil

${ }^{22}$ Universidade Federal do ABC, Santo André, SP, Brazil

${ }^{23}$ Universidade Federal do Rio de Janeiro, Instituto de Física, Rio de Janeiro, RJ, Brazil

${ }^{24}$ Universidade Federal Fluminense, EEIMVR, Volta Redonda, RJ, Brazil

${ }^{25}$ Rudjer Bošković Institute, 10000 Zagreb, Croatia

${ }^{26}$ Charles University, Faculty of Mathematics and Physics, Institute of Particle and Nuclear Physics, Prague, Czech Republic

${ }^{27}$ Institute of Physics of the Academy of Sciences of the Czech Republic, Prague, Czech Republic

${ }^{28}$ Palacky University, RCPTM, Olomouc, Czech Republic

${ }^{29}$ Institut de Physique Nucléaire d'Orsay (IPNO), Université Paris 11, CNRS-IN2P3, France

${ }^{30}$ Laboratoire de l'Accélérateur Linéaire (LAL), Université Paris 11, CNRS-IN2P3, France

${ }^{31}$ Laboratoire de Physique Nucléaire et de Hautes Energies (LPNHE), Universités Paris 6 et Paris 7, CNRS-IN2P3, Paris, France

${ }^{32}$ Laboratoire de Physique Subatomique et de Cosmologie (LPSC), Université Grenoble-Alpes, CNRS/IN2P3, France

${ }^{33}$ Station de Radioastronomie de Nançay, Observatoire de Paris, CNRS/INSU, France

${ }^{34}$ SUBATECH, École des Mines de Nantes, CNRS-IN2P3, Université de Nantes, France ${ }^{35}$ Bergische Universität Wuppertal, Wuppertal, Germany

${ }^{36}$ Karlsruhe Institute of Technology-Campus South-Institut für Experimentelle Kernphysik (IEKP), Karlsruhe, Germany

${ }^{37}$ Karlsruhe Institute of Technology-Campus North-Institut für Kernphysik, Karlsruhe, Germany

${ }^{38}$ Karlsruhe Institute of Technology — Campus North-Institut für Prozessdatenverarbeitung und Elektronik, Karlsruhe, Germany

${ }^{39}$ Max-Planck-Institut für Radioastronomie, Bonn, Germany

${ }^{40}$ RWTH Aachen University, III. Physikalisches Institut A, Aachen, Germany

${ }^{41}$ Universität Hamburg, Hamburg, Germany

${ }^{42}$ Universität Siegen, Siegen, Germany

${ }^{43}$ Università di Milano and Sezione INFN, Milan, Italy

${ }^{44}$ Università di Napoli "Federico II" and Sezione INFN, Napoli, Italy

${ }^{45}$ Università di Roma II "Tor Vergata" and Sezione INFN, Roma, Italy

${ }^{46}$ Università di Catania and Sezione INFN, Catania, Italy

${ }^{47}$ Università di Torino and Sezione INFN, Torino, Italy

${ }^{48}$ Dipartimento di Matematica e Fisica "E. De Giorgi” dell'Università del Salento and Sezione INFN, Lecce, Italy

${ }^{49}$ Dipartimento di Scienze Fisiche e Chimiche dell'Università dell'Aquila and INFN, Italy

${ }^{50}$ Gran Sasso Science Institute (INFN), L'Aquila, Italy

${ }^{51}$ Istituto di Astrofisica Spaziale e Fisica Cosmica di Palermo (INAF), Palermo, Italy

52 INFN, Laboratori Nazionali del Gran Sasso, Assergi (L'Aquila), Italy

${ }^{53}$ Osservatorio Astrofisico di Torino (INAF), Università di Torino and Sezione INFN, Torino, Italy

${ }^{54}$ Benemérita Universidad Autónoma de Puebla, Puebla, Mexico

${ }^{55}$ Centro de Investigación y de Estudios Avanzados del IPN (CINVESTAV), México, Mexico

${ }^{56}$ Universidad Michoacana de San Nicolas de Hidalgo, Morelia, Michoacan, Mexico

${ }^{57}$ Universidad Nacional Autonoma de Mexico, Mexico, D.F., Mexico

${ }^{58}$ IMAPP, Radboud University Nijmegen, Netherlands

${ }^{59} \mathrm{KVI}-$ Center for Advanced Radiation Technology, University of Groningen, Netherlands

${ }^{60}$ Nikhef, Science Park, Amsterdam, Netherlands

${ }^{61}$ ASTRON, Dwingeloo, Netherlands 
62 Institute of Nuclear Physics PAN, Krakow, Poland

${ }^{63}$ University of Łódź, Łódź, Poland

${ }^{64}$ Laboratório de Instrumentação e Física Experimental de Partículas—LIP and Instituto Superior Técnico—IST, Universidade de Lisboa—UL, Portugal

65 'Horia Hulubei' National Institute for Physics and Nuclear Engineering, Bucharest- Magurele, Romania

${ }^{66}$ Institute of Space Sciences, Bucharest, Romania

${ }^{67}$ University of Bucharest, Physics Department, Romania

${ }^{68}$ University Politehnica of Bucharest, Romania

${ }^{69}$ Experimental Particle Physics Department, J. Stefan Institute, Ljubljana, Slovenia

Laboratory for Astroparticle Physics, University of Nova Gorica, Slovenia

${ }^{71}$ Universidad Complutense de Madrid, Madrid, Spain

72 Universidad de Alcalá, Alcalá de Henares (Madrid), Spain

${ }^{73}$ Universidad de Granada and C.A.F.P.E., Granada, Spain

${ }^{74}$ Universidad de Santiago de Compostela, Spain

${ }^{75}$ School of Physics and Astronomy, University of Leeds, UK

${ }^{76}$ Case Western Reserve University, Cleveland, OH, USA

${ }^{77}$ Colorado School of Mines, Golden, CO, USA

${ }^{78}$ Colorado State University, Fort Collins, CO, USA

${ }^{79}$ Colorado State University, Pueblo, CO, USA

${ }^{80}$ Department of Physics and Astronomy, Lehman College, City University of New York, New York, USA

${ }^{81}$ Fermilab, Batavia, IL, USA

${ }^{82}$ Louisiana State University, Baton Rouge, LA, USA

${ }^{83}$ Michigan Technological University, Houghton, MI, USA

${ }_{85}^{84}$ New York University, New York, NY, USA

${ }^{85}$ Northeastern University, Boston, MA, USA

${ }^{86}$ Ohio State University, Columbus, OH, USA

${ }^{87}$ Pennsylvania State University, University Park, PA, USA

${ }^{88}$ University of Chicago, Enrico Fermi Institute, Chicago, IL, USA

${ }_{90}^{89}$ University of Hawaii, Honolulu, HI, USA

${ }^{90}$ University of Nebraska, Lincoln, NE, USA

${ }^{91}$ University of New Mexico, Albuquerque, NM, USA

Received 2014 November 15; accepted 2015 February 8; published 2015 April 24

\begin{abstract}
We analyze the distribution of arrival directions of ultra-high-energy cosmic rays recorded at the Pierre Auger Observatory in 10 years of operation. The data set, about three times larger than that used in earlier studies, includes arrival directions with zenith angles up to $80^{\circ}$, thus covering from $-90^{\circ}$ to $+45^{\circ}$ in declination. After updating the fraction of events correlating with the active galactic nuclei (AGNs) in the Véron-Cetty and Veron catalog, we subject the arrival directions of the data with energies in excess of $40 \mathrm{EeV}$ to different tests for anisotropy. We search for localized excess fluxes, self-clustering of event directions at angular scales up to $30^{\circ}$, and different threshold energies between 40 and $80 \mathrm{EeV}$. We then look for correlations of cosmic rays with celestial structures both in the Galaxy (the Galactic Center and Galactic Plane) and in the local universe (the SuperGalactic Plane). We also examine their correlation with different populations of nearby extragalactic objects: galaxies in the 2MRS catalog, AGNs detected by Swift-BAT, radio galaxies with jets, and the Centaurus A (Cen A) galaxy. None of the tests show statistically significant evidence of anisotropy. The strongest departures from isotropy (post-trial probability $\sim 1.4 \%$ ) are obtained for cosmic rays with $E>58 \mathrm{EeV}$ in rather large windows around Swift AGNs closer than $130 \mathrm{Mpc}$ and brighter than $10^{44} \mathrm{erg} \mathrm{s}^{-1}$ (18 radius), and around the direction of Cen $\mathrm{A}\left(15^{\circ}\right.$ radius $)$.
\end{abstract}

Key words: acceleration of particles - astroparticle physics

\section{INTRODUCTION}

The measurements of the energy spectrum of ultra-highenergy cosmic rays (UHECRs), their mass composition, and the celestial distribution of their arrival directions serve in a complementary way to understanding their origin. The acceleration mechanism, as well as the propagation in the Galactic and intergalactic media, can be constrained by detailed studies of spectral features and of the evolution of the mass

\footnotetext{
92 Deceased.

93 Now at Konan University.

94 Also at the Universidad Autonoma de Chiapas on leave of absence from Cinvestav.

95 Now at Unidad Profesional Interdisciplinaria de Ingeniería y Tecnologías Avanzadas del IPN, México, D.F., México.

96 Now at Universidad Autónoma de Chiapas, Tuxtla Gutiérrez, Chiapas, México.

97 Also at Vrije Universiteit Brussels, Belgium.
}

composition as a function of energy. In turn, and despite the fact that UHECRs are mostly charged particles, information on the sources might be contained in the distribution of their arrival directions, especially above a few tens of $\mathrm{EeV}$, where the magnetic deflections (at least of those cosmic rays with a small charge) may be only a few degrees. A number of facts contribute to this expectation. Stringent limits to the flux of primary photons at such energies (Abraham et al. 2009) strongly constrain top-down models for the origin of UHECRs and, hence, favor astrophysical objects as accelerators. Also, at such energies, the flux of cosmic rays is expected to be suppressed due to energy losses in their interactions with photons of the Cosmic Microwave Background by the socalled Greisen-Zatsepin-Kuz'min (GZK) effect (Greisen 1966; Zatsepin \& Kuzmin 1966). These interactions limit the distance from which a source can contribute to the flux at Earth. For instance, this distance has to be less than $\sim 200 \mathrm{Mpc}$ for protons 
or Fe nuclei with energies above $60 \mathrm{EeV}$, and even smaller for intermediate mass nuclei (Harari et al. 2006). Thus, the number of candidate sources that could contribute to the measured fluxes at the highest energies is significantly reduced. Finally, the arrival directions of UHECRs are not expected to be completely isotropized by magnetic fields due to their very high rigidity.

A suppression in the flux of UHECRs at energies above $40 \mathrm{EeV}$ has been established experimentally beyond any doubt (Abbasi et al. 2008; Abraham et al. 2008b; Abu-Zayyad et al. 2013). The energy at which the spectrum steepens is in accordance with that expected from the GZK effect. However, this alone does not allow one to conclude whether the observed feature is due to propagation effects or to source properties, i.e., the maximum energy achievable in the acceleration process. Information on the nature of UHECRs is one of the keys in discriminating between the two scenarios. The measurement of the cosmic ray composition has been addressed through the measurement of the depth of shower maximum, $X_{\max }$ (Abbasi et al. 2010, 2014b; Abraham et al. 2010a; Aab et al. 2014b). Interpretations of Auger data through the most updated models of hadronic interactions (Abreu et al. 2013b; Aab et al. 2014) indicate that the fraction of heavy nuclei increases above the energy of the ankle (the spectral hardening taking place at $E \simeq 5 \mathrm{EeV}$ ) and up to the highest energies. However, the small number of events does not allow one to probe the primary mass evolution in detail at energies in excess of $40 \mathrm{EeV}$, where there have been only 18 events available for the composition analysis.

To complement the spectrum and mass measurements, several studies of the distribution of arrival directions have been conducted with UHECR data. Using an early data set, the Pierre Auger Collaboration reported evidence of anisotropy with a confidence level of $99 \%$ in the distribution of cosmic rays with energy above about $57 \mathrm{EeV}$ (Abraham et al. 2007, 2008a). That analysis was based on the finding, through an a priori test, of a correlation within a small angular separation $(3: 1)$ between the UHECR arrival directions and the locations of nearby active galaxies (within $75 \mathrm{Mpc}$ ) in the Véron-Cetty and Véron (VCV) catalog (Veron-Cetty \& Veron 2006). With an enlarged data set, the correlating fraction was found in later analyses to be lower (Abreu et al. 2010; Kampert et al. 2012), although still $\sim 3 \sigma$ above expectations from an isotropic distribution. Other tests on the data, using a variety of astronomical catalogs, yielded some further hints but no significant evidence of anisotropy (Abreu et al. 2010). It is interesting to note that both the Pierre Auger and the Telescope Array Collaborations have reported, although with a limited significance, concentrations of very high-energy events in regions of the sky of $\sim 20^{\circ}$ radius, namely for $18^{\circ}$ around the radio galaxy Centaurus A (Cen A) in the case of Auger (Abreu et al. 2010) and in a $20^{\circ}$ radius window at declination $\delta=43^{\circ}$ in the case of the Telescope Array (Abbasi et al. 2014a). Note that the hot spot observed around Cen A is outside the field of view of the Telescope Array, while the one observed by the Telescope Array is only partially inside the field of view of the Auger Observatory when highly inclined events are considered.

In the present situation, where the origin of the suppression in the flux of the UHECRs has not yet been understood, their mass composition is not precisely known and the predictions of their deflections in magnetic fields are uncertain (also due to uncertainties in models of magnetic fields, see, for example, Farrar 2014 for a recent review), a large number of events is essential when looking for anisotropies in a sky map. Whatever the origin of the suppression in their flux and whatever their nature, UHECRs are still expected to come from sources relatively close to the Earth, where the galaxies are distributed non uniformly. Even if low-charge particles were to contribute only a fraction of the primary cosmic rays, anisotropic signals on small angular scales may show up as the number of events gathered increases. In turn, should the UHECRs be significantly deflected, either due to their large charge or the presence of strong intervening magnetic fields, directional excesses might still be found at larger angular scales. Searches for such anisotropies have so far been made with data sets including a few dozen cosmic rays (for instance, in Abreu et al. (2010) we published the arrival directions and energies of 69 events above $55 \mathrm{EeV}$ and zenith angle $\theta \leqslant 60^{\circ}$, corresponding to an exposure of $20,370 \mathrm{~km}^{2}$ sryr). In this paper we present a study of the arrival directions of UHECRs detected by the Pierre Auger Observatory in more than 10 years of data taking, with an exposure of about $66,000 \mathrm{~km}^{2}$ sryr. The data set, including more than 600 events above $40 \mathrm{EeV}$, is described in Section 2. By including for the first time cosmic rays with zenith angles up to $80^{\circ}$, the field of view of the Auger Observatory has been extended to cover from $-90^{\circ}$ to $+45^{\circ}$ in declination.

In the later sections we analyze the distribution of the arrival directions. In Section 3, we update the fraction of events correlating with active galactic nuclei (AGNs) in the VCV catalog. In spite of the large data set (three times larger than that used in Abreu et al. (2010)), this test does not substantiate the initial evidence of anisotropy at energies larger than $53 \mathrm{EeV} .{ }^{98}$ Consequently, in the later sections we explore the set of arrival directions for cosmic rays observed with energies above $40 \mathrm{EeV}$. Since this energy corresponds to the onset of the suppression in the observed flux, we expect a limited number of contributing sources above such a threshold. Also, above this energy the angular deflections caused by intervening magnetic fields are expected to be of the order of a few degrees for protons, and $Z$ times larger in the case of nuclei with atomic number $Z$. We perform various tests to search for anisotropies in the data set, exploring a wide range of angular windows between $1^{\circ}$ and $30^{\circ}$ and energy thresholds from $40 \mathrm{EeV}$ up to $80 \mathrm{EeV}$. The angular range is motivated, at the lower end, by the angular resolution of the measurement of the arrival directions and, at the higher end, by the large deflections expected if cosmic rays are high- $Z$ nuclei. Considering energy thresholds higher than $40 \mathrm{EeV}$ may help because it may involve smaller deflections and smaller GZK horizons, with the upper value of $80 \mathrm{EeV}$ still allowing a sizeable number of events (22) in the analysis. In Section 4 we study "intrinsic" anisotropies that can be revealed by the search for localized excesses of events over the exposed sky and by the analysis of the autocorrelation of arrival directions. In Section 5 we search for correlations with known astrophysical structures, such as the Galactic and Super-Galactic Planes and the Galactic Center. We study the cross-correlation with astrophysical objects that could be considered as plausible candidates for UHECR

\footnotetext{
98 This threshold was $57 \mathrm{EeV}$ in the original calibration used in Abraham et al. $(2007,2008 \mathrm{a})$. It became $55 \mathrm{EeV}$ with the updated reconstruction used in Abreu et al. (2010), corresponding to approximately $53 \mathrm{EeV}$ in the new energy scale considered in the present work (see Section 2).
} 
sources in Section 6. Specifically, we exploit flux-limited catalogs of galaxies (2MRS), of AGNs observed in X-rays (Swift BAT-70), and of radio galaxies with jets. For the last two samples, we perform an additional study, considering different thresholds in the AGN intrinsic luminosity. Finally, in Section 7 we focus on the distribution of events around the direction of Cen A. After summarizing the main results in Section 8 we report in the Appendix the list of arrival directions and energies of the 231 UHECRs with energies above $52 \mathrm{EeV}$ detected by the Pierre Auger Observatory between 2004 January 1 and 2014 March $31 .^{99}$

\section{THE DATA SET}

The Pierre Auger Observatory (Abraham et al. 2004) is located in Malargüe, Argentina, at latitude 35:2 S, longitude $69.5 \mathrm{~W}$, and an average altitude of $1400 \mathrm{~m}$ a.s.l. It comprises a surface detector (SD) made up of an array of water-Cherenkov stations overlooked by an air-fluorescence detector (FD) comprising a total of 27 telescopes at four sites on the perimeter of the array. The array consists of 1,660 waterCherenkov stations covering an area of about $3000 \mathrm{~km}^{2}$. The SD samples the particle components of extensive air showers (mainly muons, electrons, and photons) with a duty cycle of nearly $100 \%$.

The data set analyzed here includes cosmic rays with energy above $40 \mathrm{EeV}$ recorded by the SD from 2004 January 1 up to 2014 March 31. In earlier analyses of the arrival directions we have used events with zenith angles less than $60^{\circ}$ (referred to as vertical). Here we include, for the first time, those with zenith angles from $60^{\circ}$ up to $80^{\circ}$ (dubbed inclined). Selection, reconstruction, and energy determination are different for the two event sets. The main characteristics of the data sets, including energy and angular resolution, are outlined below, and details can be found in Ave et al. (2007) and Aab et al. (2014a).

Vertical events are accepted if at least four of the closest stations to the one with the highest signal are operational at the time of the event. We also require that the reconstructed shower core be contained within a triangle, either equilateral or isosceles, of contiguous operational stations. This event selection, a less stringent one than that used in earlier works (where five operational neighboring stations were required), has been carefully studied using data. It ensures an accurate event reconstruction given the large multiplicity of triggered detectors (on average more than 14 stations are triggered in events with energy above $40 \mathrm{EeV}$ ). It also allows us to increase the number of vertical events by about $14 \%$ in the period considered, a value consitent with the increase in aperture gained with the more relaxed trigger. On the other hand, for inclined events, we require that at least five active stations surround the station closest to the core position. Given the large footprint of inclined showers on the ground (the average station multiplicity is larger than 30), such a fiducial criterion guarantees adequate containment inside the array. The described selections lead to 454 vertical and 148 inclined events with $E \geqslant 40 \mathrm{EeV}$.

The trigger and selection efficiency is $100 \%$ for energies above $3 \mathrm{EeV}$ for vertical showers and $4 \mathrm{EeV}$ for inclined showers. The exposure is consequently determined by purely

\footnotetext{
99 The list of the events is also available at http://auger.org/data/ AugerUHECR2014.txt.
}

geometrical considerations (Abraham et al. 2010b; Aab et al. 2013) in both cases, and for the period considered here it amounts to 51,753 and $14,699 \mathrm{~km}^{2}$ sr yr, for the vertical and inclined samples, respectively.

For both data sets, the arrival directions of cosmic rays are determined from the relative arrival times of the shower front in the triggered stations. The angular resolution, defined as the radius around the true cosmic ray direction that would contain $68 \%$ of the reconstructed shower directions, is better than $0: 9$ for energies above $10 \mathrm{EeV}$ (Bonifazi et al. 2009).

The ground parameters used to estimate the primary energy are different for the two data sets. The estimator for the primary energy of vertical showers is the reconstructed signal at $1000 \mathrm{~m}$ from the shower axis, denoted $S(1000)$. The energy reconstruction of an inclined shower is based on the muon content, denoted $N_{19}$, relative to a simulated proton shower with energy $10^{19} \mathrm{eV}$. In both cases, the energy estimators are calibrated using hybrid events (detected simultaneously by SD and FD) and using the quasi-calorimetric energy determination obtained with the air-fluorescence detector (Aab et al. 2013, 2014a). The statistical uncertainty in the energy determination is smaller than $12 \%$ for energies above $10 \mathrm{EeV}$ (Abreu et al. 2011; Aab et al. 2014a). The systematic uncertainty in the absolute energy scale, common to the two data sets, is $14 \%$. The Pierre Auger Collaboration has updated the energy scale in Verzi et al. (2013) accounting for recent measurements of the fluorescence yield (Ave et al. 2013), a better estimate of the invisible energy (Tueros et al. 2013), a deeper understanding of the detector, and an improved event reconstruction. The energy threshold of $55 \mathrm{EeV}$ used in our previous publication (Abreu et al. 2010) now corresponds to approximately $53 \mathrm{EeV}$ with the new energy scale.

We note that the relative number of vertical and inclined events above $40 \mathrm{EeV}, 454 / 148 \simeq 3.07 \pm 0.29$, is consistent in view of the Poissonian fluctuations with the corresponding ratio of exposures, $51,753 / 14,699 \simeq 3.52$. On the other hand, the $14 \%$ difference between these ratios could also result from a $4 \%$ mismatch between the vertical and inclined energy calibrations, which is compatible with the uncorrelated systematic uncertainties on the energy scale.

\section{NOTE ON THE ANISOTROPY TEST WITH THE VCV CATALOG}

One of the anisotropy tests performed in our previous works was based on the VCV catalog of AGNs (Veron-Cetty \& Veron 2006). In an initial study, we considered vertical events with $E \geqslant 40 \mathrm{EeV}$ collected from 2004 January 1 to 2006 May 26 (Period I). We performed an exploratory scan over the energy threshold of the events, their angular separation from AGNs, and the maximum AGN redshift. We found that the most significant excess appeared in the correlation of events with energy above $57 \mathrm{EeV}$ and lying within 3:1 of those AGNs closer than $75 \mathrm{Mpc}$. These parameters were then used for a search on independent data, where it was found that 8 out of 13 events correlated, while 2.7 events (i.e., $21 \%$ of the total) were expected to correlate by chance for an isotropic distribution of arrival directions. This finding had a probability of $1.7 \times 10^{-3}$ of happening by chance (Abraham et al. 2007, 2008a). Subsequent analyses with enlarged statistics yielded a correlation that was still above isotropic expectations, but with a smaller strength and essentially dominated by the initial excess. The 


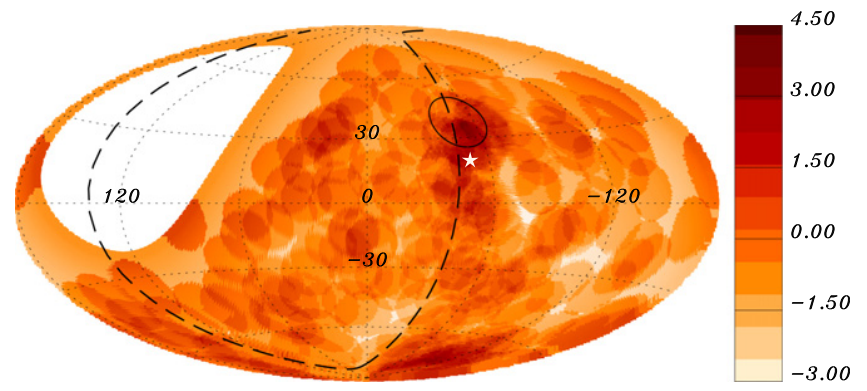

Figure 1. Map in Galactic coordinates of the Li-Ma significances of overdensities in $12^{\circ}$-radius windows for the events with $E \geqslant 54 \mathrm{EeV}$. Also indicated are the Super-Galactic Plane (dashed line) and Centaurus A (white star).

level of correlation was $\left(38_{-6}^{+7}\right) \%$ in Abreu et al. (2010) and $(33 \pm 5) \%$ in Kampert et al. (2012).

Here we update this analysis, for historical reasons, by using the vertical data set described in Section 2 and the VCV catalog used in Abraham et al. (2007). Excluding Period I, there are 146 events above $53 \mathrm{EeV}$ : 41 events correlate with VCV AGNs, with the angular and distance parameters fixed by the exploratory scan. The updated fraction of correlations is then $\left(28.1_{-3.6}^{+3.8}\right) \%$, which is two standard deviations above the isotropic expectation of $21 \%$. On the other hand, note that since the VCV correlations involve many different regions of the sky (besides the fact that CRs with different energies have significant time delays), so an explanation of the reduced correlation found after 2007 in terms of a transient nature of the signal would not be natural. Hence, the high level of correlation found initially was probably affected by a statistical fluctuation. We conclude that this particular test does not yield a significant indication of anisotropy with the present data set.

\section{GENERAL ANISOTROPY TESTS}

\subsection{Search for a Localized Excess Flux over the Exposed Sky}

A direct analysis of cosmic ray arrival directions is the blind search for excesses of events over the visible sky. To this aim, we sample the exposed sky using circular windows with radii varying from $1^{\circ}$ up to $30^{\circ}$, in $1^{\circ}$ steps. The centers of the windows are taken on a $1^{\circ} \times 1^{\circ}$ grid. The energy threshold of the events used to build the maps is varied from $40 \mathrm{EeV}$ up to $80 \mathrm{EeV}$ in steps of $1 \mathrm{EeV}$. To detect an excess, for every window and energy threshold we compare the number of observed events, $n_{\mathrm{obs}}$, with that expected from an isotropic flux of cosmic rays, $n_{\text {exp }}$. For each sky direction, the expected number of events for an isotropic distribution is obtained by numerically integrating the geometric exposures in the corresponding windows. We use the total number of vertical and inclined events to normalize the relative exposures of the two samples. Note that since the triggering is different in the two cases, this fraction is non-trivial.

For each window, we calculate the binomial probability, $p$, of observing by chance in an isotropic flux an equal, or larger, number of events than that found in the data. We find the minimum probability, $p=5.9 \times 10^{-6}$, at an energy threshold of $54 \mathrm{EeV}$ and in a $12^{\circ}$-radius window centered at right ascension and declination $(\alpha, \delta)=\left(198^{\circ},-25^{\circ}\right)$, i.e., for Galactic longitude and latitude $(\ell, b)=\left(-51^{\circ} .1,37^{\circ} .6\right)$, for which $n_{\text {obs }} / n_{\text {exp }}=14 / 3.23$. The map of the Li-Ma ( $\mathrm{Li} \&$ Ma 1983) significances of the excesses of events with $E \geqslant 54$
$\mathrm{EeV}$ in windows of $12^{\circ}$ radius is shown in Figure 1. The highest significance region just discussed, having a $\mathrm{Li}-\mathrm{Ma}$ significance of $4.3 \sigma$, is indicated with a black circle. It is close to the Super-Galactic Plane, indicated with a dashed line, and centered at about $18^{\circ}$ from the direction of Cen A, indicated with a white star. One should note that although the effect of a turbulent magnetic field would just be to spread a signal around the direction toward the source, a regular field that is coherent over large scales would give rise to a shift in the excess in a direction orthogonal to that of the magnetic field, with the size of both effects being energy dependent.

To assess the significance of this excess, we simulated 10,000 sets of isotropic arrival directions containing the same number of events as the data set. In doing so, we keep the original energies of the events and assign to them random arrival directions according to the geometric exposure, choosing randomly between vertical and inclined events according to their relative exposures. We apply to the simulated sets the same scans in angle and energy as those applied to the data. We find that values smaller than $p=5.9 \times 10^{-6}$ are obtained in $69 \%$ of isotropic simulations, and hence the excess found in the data turns out to be compatible with the maximum excesses expected in isotropic simulations. We note that in the region of the hot spot reported by the Telescope Array Collaboration (Abbasi et al. 2014a), a 20 ${ }^{\circ}$ radius circular window centered at $(\alpha, \delta)=\left(146^{\circ} .7,43^{\circ} .2\right)$ which is partially outside our field of view, we would expect to see 0.97 events with $E>53 \mathrm{EeV}$ if the distribution were isotropic; one event is observed.

\subsection{The Autocorrelation of Events}

Another simple way to test the clustering of arrival directions is through an autocorrelation analysis, which is particularly useful when several sources lead to excesses around them on a similar angular scale. With this method, one looks for excesses in the number of pairs of events, i.e., excesses of "selfclustering," namely, we count the number of pairs of events, $N_{\mathrm{p}}\left(\psi, E_{\mathrm{th}}\right)$, above a given energy threshold, $E_{\mathrm{th}}$, that are within a certain angular distance, $\psi$. We do this at different energy thresholds, from 40 up to $80 \mathrm{EeV}$ (in steps of $1 \mathrm{EeV}$ ) and we look at angular scales from $1^{\circ}$ up to $30^{\circ}$ (in steps of 0.25 up to $5^{\circ}$, and of $1^{\circ}$ for larger angles). To identify an excess, we compare the observed number of pairs with that expected from an isotropic distribution having the same number of arrival directions above the corresponding energy threshold. For each energy threshold and angle we then calculate the fraction of isotropic simulations having an equal number of, or more pairs than the data, $f\left(\psi, E_{\mathrm{th}}\right)$.

The result is shown in Figure 2 as a function of the angular distance and the energy threshold. The color code indicates the values obtained for $f$. The white cross corresponds to the parameter values leading to the minimum value of this fraction, $f_{\text {min }}=0.027$, which happens for $\psi=1.5$ and $E_{\text {th }}=42 \mathrm{EeV}$. For these parameters, 30 pairs are expected, on average, for isotropic simulations, while 41 are observed in the data. We calculate the post-trial probability for this excess, $P$, as the fraction of isotropic simulations that under a similar scan over $E_{\text {th }}$ and $\psi$ lead to a value of $f_{\min }$ smaller than the one obtained with the data. The resulting value, $P \simeq 70 \%$, indicates that the autocorrelation is compatible with the expectations from an isotropic distribution of arrival directions. 


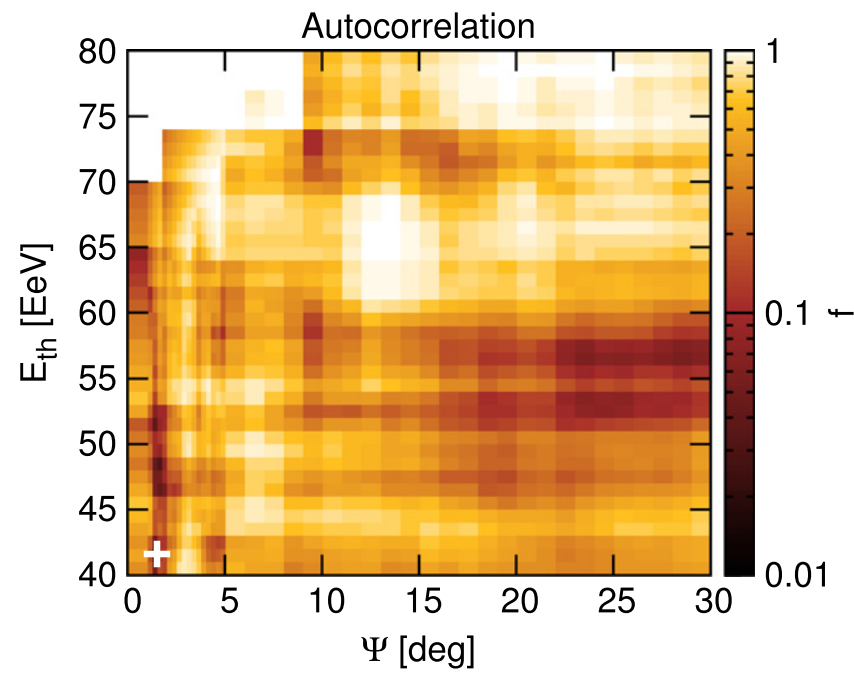

Figure 2. Fraction $f$ obtained in the autocorrelation of events vs. $\psi$ and $E_{\mathrm{th}}$.

\section{SEARCH FOR CORRELATIONS WITH THE GALAXY AND WITH THE SUPER-GALACTIC PLANE}

In the previous section, we tested the intrinsic distribution of arrival directions of UHECRs, i.e., without formulating any hypothesis on the distribution of their sources. In the following, we consider specific astrophysical structures and objects as candidate sources. In this section, we search for correlations with the Galactic and the Super-Galactic Planes as well as with the Galactic Center. On the one hand, a Galactic origin of UHECRs might give rise to an excess of arrival directions near the plane of the Galaxy, especially if a low- $Z$ primary component contributes to the CR flux. On the other hand, nearby galaxies (within $100 \mathrm{Mpc}$ ) show a clustering along the so-called Super-Galactic Plane, which contains several prominent (super) clusters such as Virgo, Centaurus, Norma, PavoIndus, Perseus-Pisces, Coma, etc., and, hence, extragalactic cosmic rays could be clustered near the Super-Galactic Plane.

We search for excesses of events as a function of Galactic (Super-Galactic) latitude, $b_{\mathrm{G}}\left(b_{\mathrm{SG}}\right)$, considering different latitude bands, $|b|<b(\max )$, with $b(\max )$ indicating the half-width of the band. To identify an excess, we compare the number of events observed within the latitude band considered with those obtained in isotropic simulations for the distribution of arrival directions. The plots in Figure 3 display the fraction, $f$, of isotropic simulations leading to a larger number of events than the data for the different energy thresholds and angular scales considered. The left figure represents the excesses in different latitude bands around the Galactic Plane, leading to a minimum value of $f_{\min } \simeq 0.05$ for $E \geqslant 72 \mathrm{EeV}$ and $b_{\mathrm{G}}(\max )=30^{\circ}$, indicated with a white cross in the figure. In this case, 29 events are observed, but 22.8 would be expected on average in isotropic simulations. The penalized probability for obtaining a smaller value of $f_{\min }$ in isotropic simulations after a similar scan is $P=70 \%$. The right plot is similar but represents the excesses in different super-galactic latitude bands, leading to a minimum value of $f_{\min } \simeq 0.035$ for $E \geqslant 53 \mathrm{EeV}$ and $b_{\mathrm{SG}}(\max )=19^{\circ}$. In this case, 89 events are observed, but 69.7 would be expected on average in isotropic simulations. The penalized probability for obtaining a smaller value of $f_{\min }$ in isotropic simulations after a similar scan is $P=22 \%$.

The corresponding results for circular windows around the Galactic Center are shown in Figure 4. The minimum, $f_{\min }$, is obtained for an angular radius around the GC of $19^{\circ}$ and for $E \geqslant 60 \mathrm{EeV}$, for which 12 events are observed while 5.6 are expected on average for isotropic simulations. The penalized probability in this case is $P \simeq 29 \%$, so that no significant excess results for any of the cases considered in this section.

\section{SEARCH FOR CROSS-CORRELATIONS WITH ASTROPHYSICAL CATALOGS}

In this section, we search for correlations of the set of arrival directions with the celestial distribution of potential nearby cosmic ray sources. We choose approximately uniform and complete catalogs, namely the 2MRS catalog of galaxies (Huchra et al. 2012), the Swift-BAT (Baumgartner et al. 2013) $\mathrm{X}$-ray catalog of $\mathrm{AGNs}^{100}$, and a catalog of radio galaxies with jets recently compiled in Van Velzen et al. (2012). The three samples are quite complementary in identifying possible cosmic ray sources. The normal galaxies that dominate the 2MRS catalog may trace the locations of gamma-ray bursts and/or fast-spinning newborn pulsars, whereas X-rays observed by Swift identify AGNs hosted mainly by spiral galaxies, and the radio emission catalog selects extended jets and radio lobes of AGNs hosted mainly by elliptical galaxies.

The 2MASS Redshift Survey (2MRS; Huchra et al. 2012) maps the distribution of galaxies in the nearby universe. It covers $91 \%$ of the sky, except for Galactic latitudes $|b|<5^{\circ}$ (and $|b|<8^{\circ}$ for longitudes within $30^{\circ}$ of the Galactic Center). In the region covered, it is essentially complete (at $97.6 \%$ ) for magnitudes brighter than $K_{s}=11.75$. It contains 43,533 galaxies with measured redshift ${ }^{101}: 37,209$ of them are within $200 \mathrm{Mpc}$ and 16,422 are within $100 \mathrm{Mpc}$. About $90 \%$ of its objects have a redshift $z<0.05$, which is the range of distances of interest for UHECR correlation studies due to the effects of the GZK horizon.

The Swift-BAT 70 months X-ray catalog (Baumgartner et al. 2013) includes sources detected in 70 months of observation of the BAT hard X-ray detector on the Swift gamma-ray burst observatory. It contains a total of 1210 objects: 705 of them are AGN-like (Seyfert I and II, other AGNs, blazars, and QSOs) with measured redshift. The catalog is complete over $90 \%$ of the sky for fluxes $>13.4 \times 10^{-12}$ $\mathrm{erg}\left(\mathrm{s} \mathrm{cm}^{2}\right)^{-1}$, measured in the X-ray band from 14 to $195 \mathrm{keV}$ (note that the completeness of the subsample of AGNs with measured redshifts maybe slightly different). We use this cut in flux hereafter to have a more uniform sample of nearby AGNs. 489 AGN-like objects survive the cut: 296 of them are within $200 \mathrm{Mpc}$ and 160 are within $100 \mathrm{Mpc}$.

The third catalog we use is a compilation of radio galaxies produced in Van Velzen et al. (2012). This is a combination of catalogs of observations at $1.4 \mathrm{GHz}$ (NRAO VLA Sky Survey (Condon et al. 1998)) and $843 \mathrm{MHz}$ (Sydney University Molonglo Sky Survey (Mauch et al. 2003)), with redshifts of associated objects taken from 2MRS. A flux limit of $213 \mathrm{mJy}$ $(289 \mathrm{mJy})$ at $1.4 \mathrm{GHz}(843 \mathrm{MHz})$ is imposed on the objects from each respective catalog, which would correspond to the flux of Cen A as seen from a distance of about $200 \mathrm{Mpc}$. We select from this catalog the radio galaxies having jets, which constitute a set of attractive candidates for UHECR sources.

\footnotetext{
100 The 2MRS and Swift catalogs have been updated after our earlier study of correlations in Abreu et al. (2010) and Kampert et al. (2012).

${ }^{101} \mathrm{We}$ adopt hereafter a Hubble constant of $H_{0}=70 \mathrm{~km} \mathrm{~s}^{-1} \mathrm{Mpc}^{-1}$ and the effective distances considered are taken as $D \equiv z c / H_{0}$, with $z$ being the source redshift obtained from the catalog.
} 

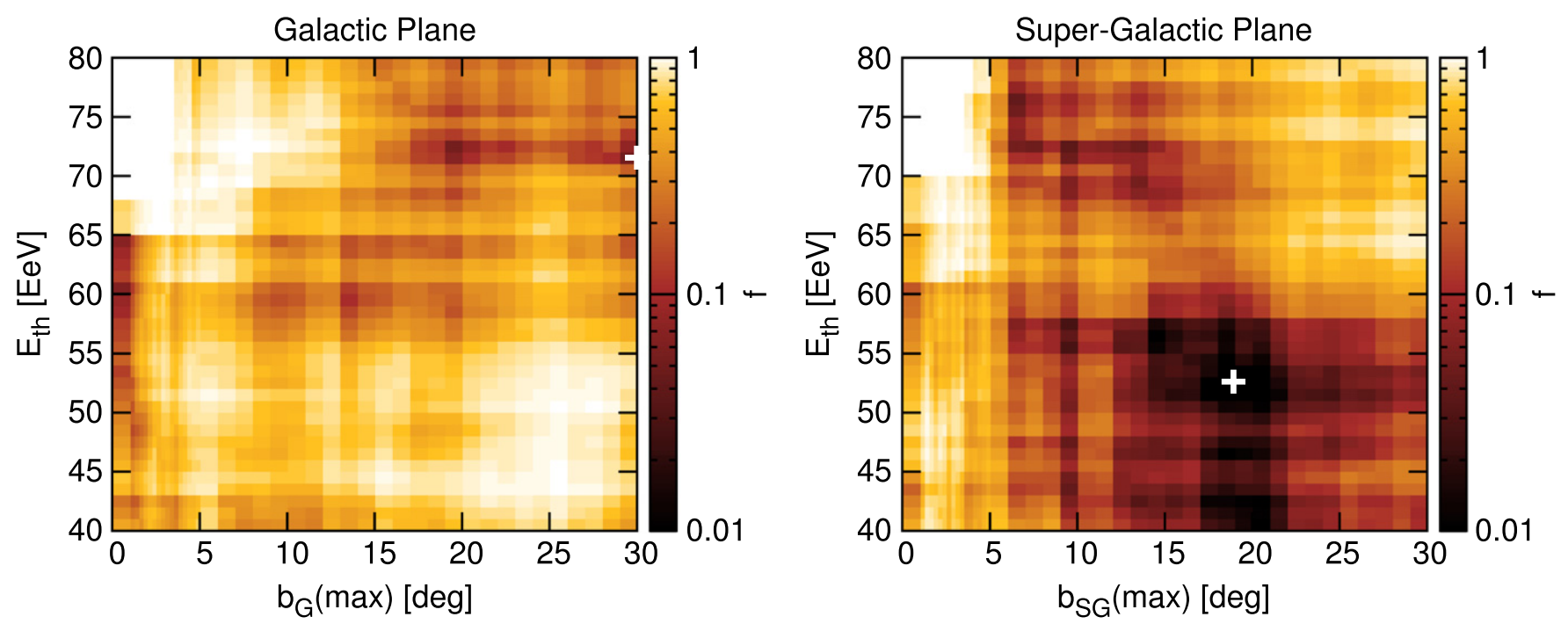

Figure 3. Fraction $f$ as a function of Galactic (left) or Super-Galactic (right) latitude band half-width considered, for events with energies above $E_{\mathrm{th}}$.

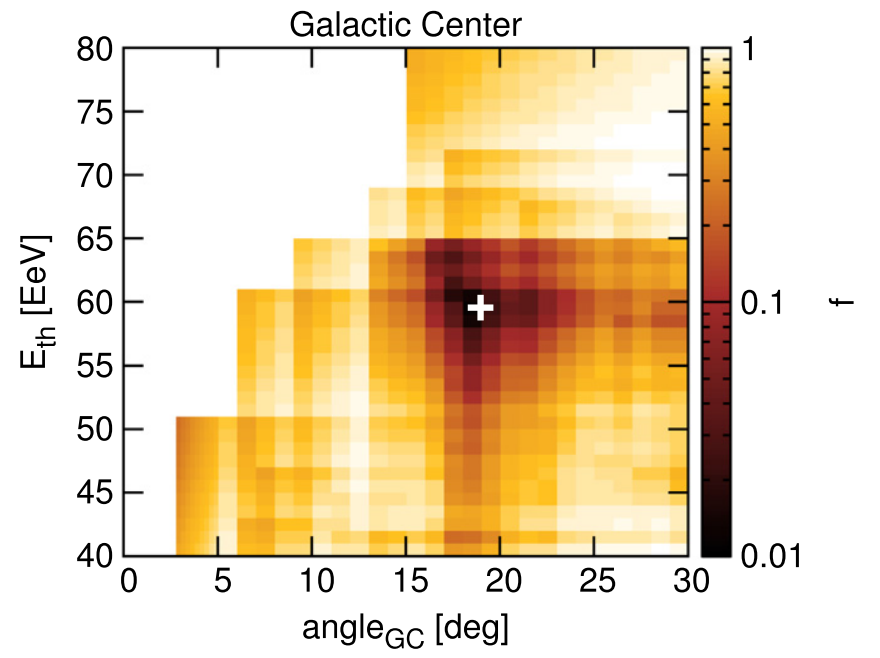

Figure 4. Fraction $f$ in circular windows around the Galactic Center as a function of the angular radius of the window and $E_{\mathrm{th}}$.

There are a total of 407 such jetted radio galaxies: 205 are within $200 \mathrm{Mpc}$ and 56 are within $100 \mathrm{Mpc}$ (for this catalog we compute the distance using the redshift corrected for peculiar velocities that are also provided). We note that the majority of these radio galaxies are different from the Swift-BAT AGNs detected in X-rays, with an overlap of only about $5 \%$. It is also important to keep in mind that although we analyze each catalog individually, it is possible that different types of sources (i.e., from different catalogs) might be contributing to the overall UHECR fluxes.

Below, we first study the cross-correlation with the three flux-limited catalogs (with the flux limits just described), including objects up to different maximum distances. This selection is based on the apparent luminosity, and is motivated by the fact that nearby sources may contribute significantly to the fluxes (in their corresponding electromagnetic band as well as in CRs) even if they are intrinsically fainter than far away sources. In the case of the AGNs in the Swift and radio-galaxy catalogs we also scan on the measured intrinsic luminosity of the objects. This is motivated by the fact that the maximum CR energy, $E_{\max }$, achievable at the sources may be linked to the intrinsic electromagnetic bolometric luminosity, $\mathcal{L}$, of the source. In particular, one could expect that $\left(E_{\max } / Z\right)^{2} \propto \mathcal{L}$ if the energy density in the magnetic field is in equipartition with the energy in synchrotron emitting electrons in the acceleration region (see, e.g., Farrar \& Gruzinov 2009). Hence, it might happen that only sources intrinsically brighter than some given luminosity are able to accelerate CRs above the threshold energies considered in this paper. On the other hand, for the radio galaxies, the luminosity is also correlated with the Fanaroff-Riley class, with FRII galaxies being generally brighter than FRI ones.

\subsection{Cross-correlation with Flux-limited Samples}

The basis of the cross-correlation technique is a counting of the number of pairs between UHE events and objects in the chosen catalogs. In a similar way to the analyses described in previous sections, we scan over energy threshold $\left(40 \mathrm{EeV} \leqslant E_{\text {th }} \leqslant 80 \mathrm{EeV}\right)$ and over the angular scale $\left(1^{\circ} \leqslant \psi \leqslant 30^{\circ}\right)$. We also consider different maximum distances to the objects, $D$, scanning on this from $10 \mathrm{Mpc}$ up to $200 \mathrm{Mpc}$, in steps of $10 \mathrm{Mpc}$. To find excesses of pairs, we compare their observed number with that resulting from isotropic simulations. For each considered distance, $D$, we first calculate the fraction of isotropic simulations having an equal number to or more pairs than the data, $f\left(\psi, E_{\mathrm{th}}\right)$, and then we look for its minimum, $f_{\min }$. The post-trial probability, $P$, is calculated as the fraction of isotropic simulations which, under similar scans over $E_{\text {th }}$ and $\psi$ for each considered $D$, lead to a value of $f_{\min }$ smaller than the one obtained with the data.

Figure 5 displays the results for the case of the 2MRS catalog. The top-left panel shows $f_{\min }$ (asterisks) and $P$ (squares) obtained for each distance, $D$. The minimum values are observed for $D=90 \mathrm{Mpc}$, for which $f_{\min } \simeq 1.5 \times 10^{-3}$ and $P \simeq 8 \%$. The top-right panel in the figure shows the distribution of $f\left(\psi, E_{\mathrm{th}}\right)$ as a function of energy threshold and angle for the value $D=90 \mathrm{Mpc}$, giving rise to the minimum probability. The local minimum (indicated with a cross) is observed for $\psi=9^{\circ}$ and $E_{\text {th }}=52 \mathrm{EeV}$. For these values, 20,042 pairs are expected on average from isotropic realizations, while 23,101 are observed in the data. Considering the penalization due to the scan in $D$ performed, the probability, $\mathcal{P}$, 

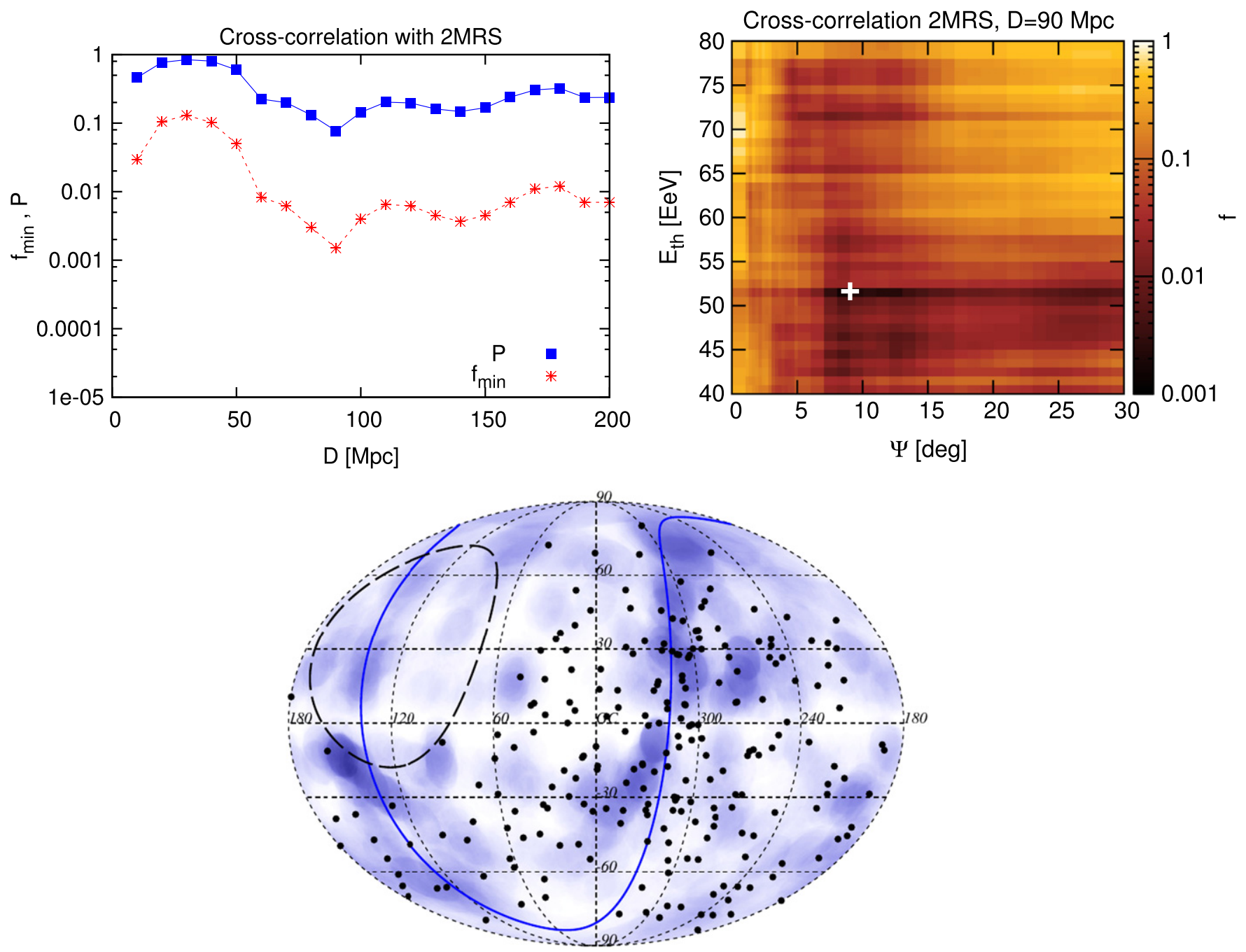

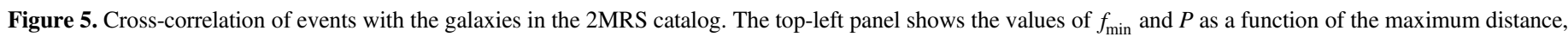

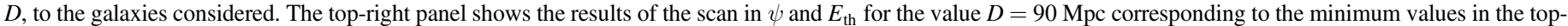

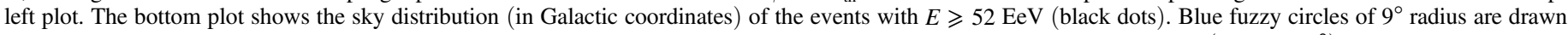

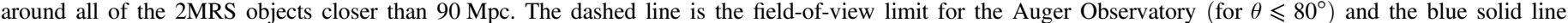
corresponds to the Super-Galactic Plane.

of obtaining a value of $P$ smaller than $8 \%$ from isotropic distributions for any value of $D$ is $\mathcal{P} \simeq 24 \%$. Finally, the bottom panel of the figure displays the map of the events with $E \geqslant 52 \mathrm{EeV}$ (black dots). Also drawn is a blue fuzzy circle around each $2 \mathrm{MRS}$ galaxy closer than $90 \mathrm{Mpc}$. All of those circles have radius $9^{\circ}$, which is the value for which the crosscorrelation has maximum significance. Given the very large number of galaxies in $2 \mathrm{MRS}$, essentially all events are within $9^{\circ}$ of at least one galaxy. Events falling in regions of the plot with denser color will have more galaxies within $9^{\circ}$ and, hence, will contribute more pairs to the cross-correlation at this angular scale.

Similar plots to those presented above are included in Figure 6 for the case of the Swift-BAT catalog. As shown in the top-left panel of the figure, the minimum values are reached here for $D=80 \mathrm{Mpc}$, where $f_{\min } \simeq 6 \times 10^{-5}$ and $P \simeq 1 \%$. Correspondingly, the top-right panel in the figure shows $f\left(\psi, E_{\text {th }}\right)$ as a function of energy and angle at $D=80 \mathrm{Mpc}$. The local minimum (indicated with a cross) is at $\psi=1^{\circ}$ and $E_{\text {th }}=58 \mathrm{EeV}$, where nine pairs are observed and 1.6 are expected, on average. After accounting for the penalization due to the scan performed in $D$, the probability of obtaining a value of $P$ smaller than $1 \%$ from isotropic distributions for any value of $D$ is $\mathcal{P} \simeq 6 \%$. Finally, we show the map of events and objects in the bottom panel. Given the minimum found, we include events with $E \geqslant 58 \mathrm{EeV}$ and draw circles of $1^{\circ}$ radius around the BAT AGNs closer than $80 \mathrm{Mpc}$.

The results of the cross-correlation with jetted radio galaxies are shown in Figure 7. The minimum value $f_{\min } \simeq 2 \times 10^{-4}$, with $P \simeq 1.4 \%$, is obtained for $D=10 \mathrm{Mpc}$ (see top-left panel). The only object included in this catalog within such a distance is the Cen A galaxy. Since the correlation with Cen A is discussed separately in the next section, we consider here the second minimum, which is found for $D=90 \mathrm{Mpc}$. This minimum corresponds to $f_{\min } \simeq 4 \times 10^{-4}$ and $P \simeq 3.4 \%$. Thus, the top-right panel in the figure shows the results of the scan in angle and energy for $D=90 \mathrm{Mpc}$. The minimum occurs for $\psi=4.75$ and $E_{\mathrm{th}}=72 \mathrm{EeV}$, where 13 pairs are observed in the data and 3.2 are expected, on average. The chance probability for getting $P \leqslant 1.4 \%$ (corresponding to the absolute minimum found) for any value of $D$ is $\mathcal{P} \simeq 8 \%$. As was done for the other catalogs, the bottom panel displays the 

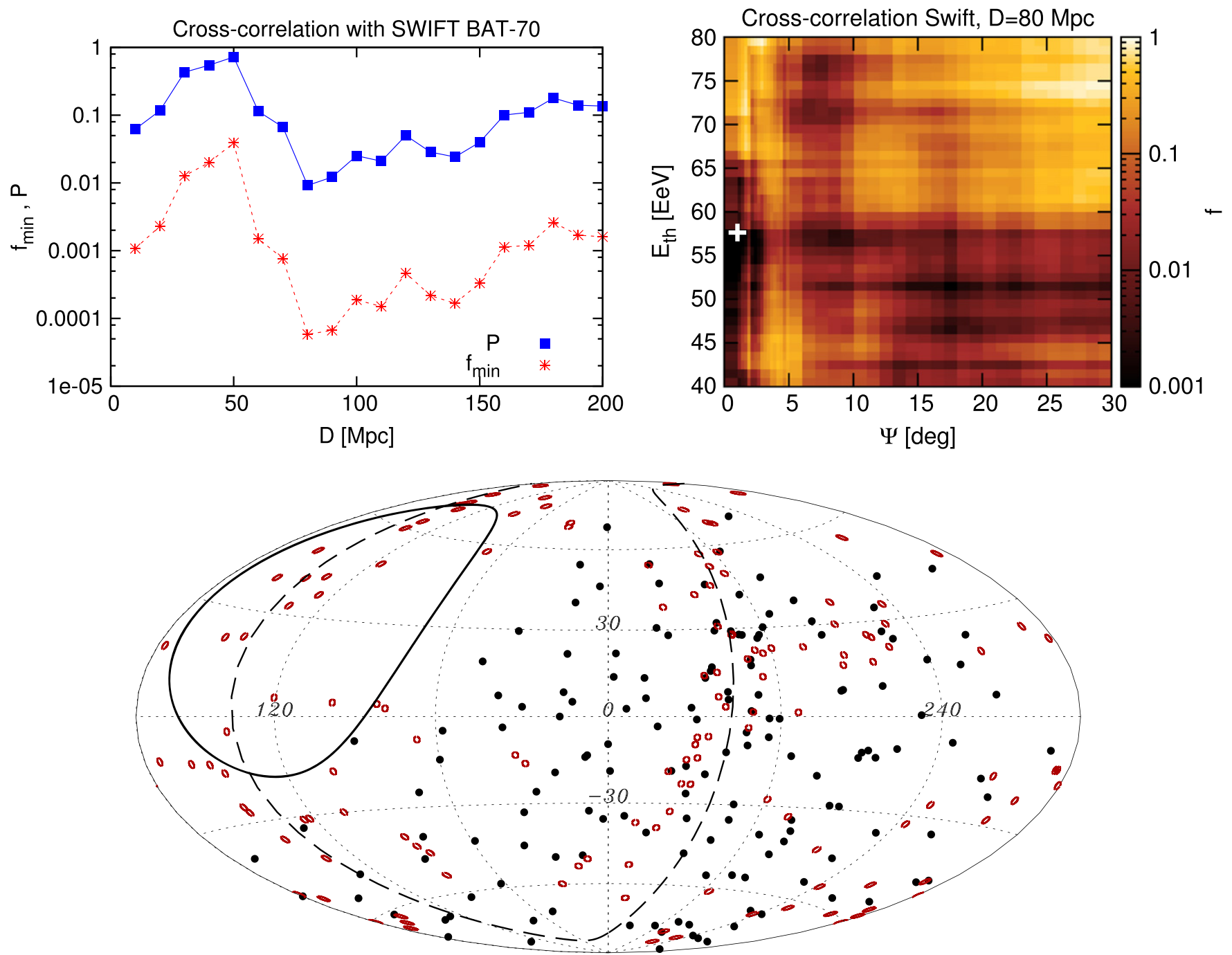

Figure 6. Cross-correlation of events with the AGNs in the Swift-BAT catalog. The top-left panel shows the values of $f_{\min }$ and $P$ as a function of the maximum distance, $D$, to the AGNs considered. The top-right panel shows the results of the scan in $\psi$ and $E_{\text {th }}$ for the value $D=80$ Mpc corresponding to the minimum values in the top-left plot. The bottom plot shows the sky distribution (in Galactic coordinates) of the events with $E \geqslant 58 \mathrm{EeV}$ (black dots). Red circles of $1^{\circ}$ radius are drawn around the BAT AGNs closer than $80 \mathrm{Mpc}$.

map of events and objects corresponding to the minimum found, i.e., $E \geqslant 72 \mathrm{EeV}$ and $D=90 \mathrm{Mpc}$. Circles of radius 4.75 are drawn around every radio galaxy and the events are indicated with black dots.

While the cross-correlation analysis does not provide us with a significant indication of excesses of pairs with any of the catalogs considered, at any energy, distance, and angle, we note that they all yield minima for similar maximum distances to the objects $(\sim 80-90 \mathrm{Mpc})$, although for different threshold energies and angular scales. The fact that the distances are similar for the three catalogs is actually expected given the existing correlations between catalogs, since AGNs are preferentially located in regions with a high density of galaxies. On the other hand, the preference toward $D \simeq 80 \mathrm{Mpc}$ is mostly due to the fact that, for this value, the whole Centaurus Supercluster gets included and in this region there is an excess of high-energy events.

\subsection{Cross-correlation with Bright AGNs}

We present here the results of a scan over the minimum source luminosities, considering for the Swift AGNs the reported X-ray band luminosity, $\mathcal{L}_{\mathrm{X}}$, and for the radio-galaxy sample the reported radio luminosity, $\mathcal{L}_{\mathrm{R}}$, computed per logarithmic energy bin at $1.1 \mathrm{GHz}$. For Swift, we scan from $\mathcal{L}_{\mathrm{X}}=10^{42}$ up to $10^{44} \mathrm{erg} \mathrm{s}^{-1}$, while for the radio galaxies we scan from $\mathcal{L}_{\mathrm{R}}=10^{39}$ up to $10^{41} \mathrm{erg} \mathrm{s}^{-1}$, considering three logarithmic steps per decade, for a total of seven luminosity values in each case. These luminosity values cover most of the range spanned by the actual luminosities of the AGNs that are present in the catalogs (just 10 AGNs from the Swift sample have $\mathcal{L}_{\mathrm{X}}<10^{42} \mathrm{erg} \mathrm{s}^{-1}$, while only three AGNs from the radio-galaxy sample have $\mathcal{L}_{\mathrm{R}}<10^{39} \mathrm{erg} \mathrm{s}^{-1}$ ). Given the additional scan performed in $\mathcal{L}$, we do a slightly coarser scan in $D$, using $20 \mathrm{Mpc}$ steps to cover from 10 up to $190 \mathrm{Mpc}$.

Considering first the Swift catalog, we show in the top-left panel of Figure 8 the resulting values of $f_{\min }$ as a function of the maximum AGN distance and the minimum adopted luminosity, $\mathcal{L}_{\text {min }}$, in the respective bands (the white region in the top-left corner of the plot is due to the absence of nearby objects above those luminosity thresholds). The values of $f_{\min }$ are obtained after scanning on $\Psi$ and $E_{\mathrm{th}}$, as in the previous 

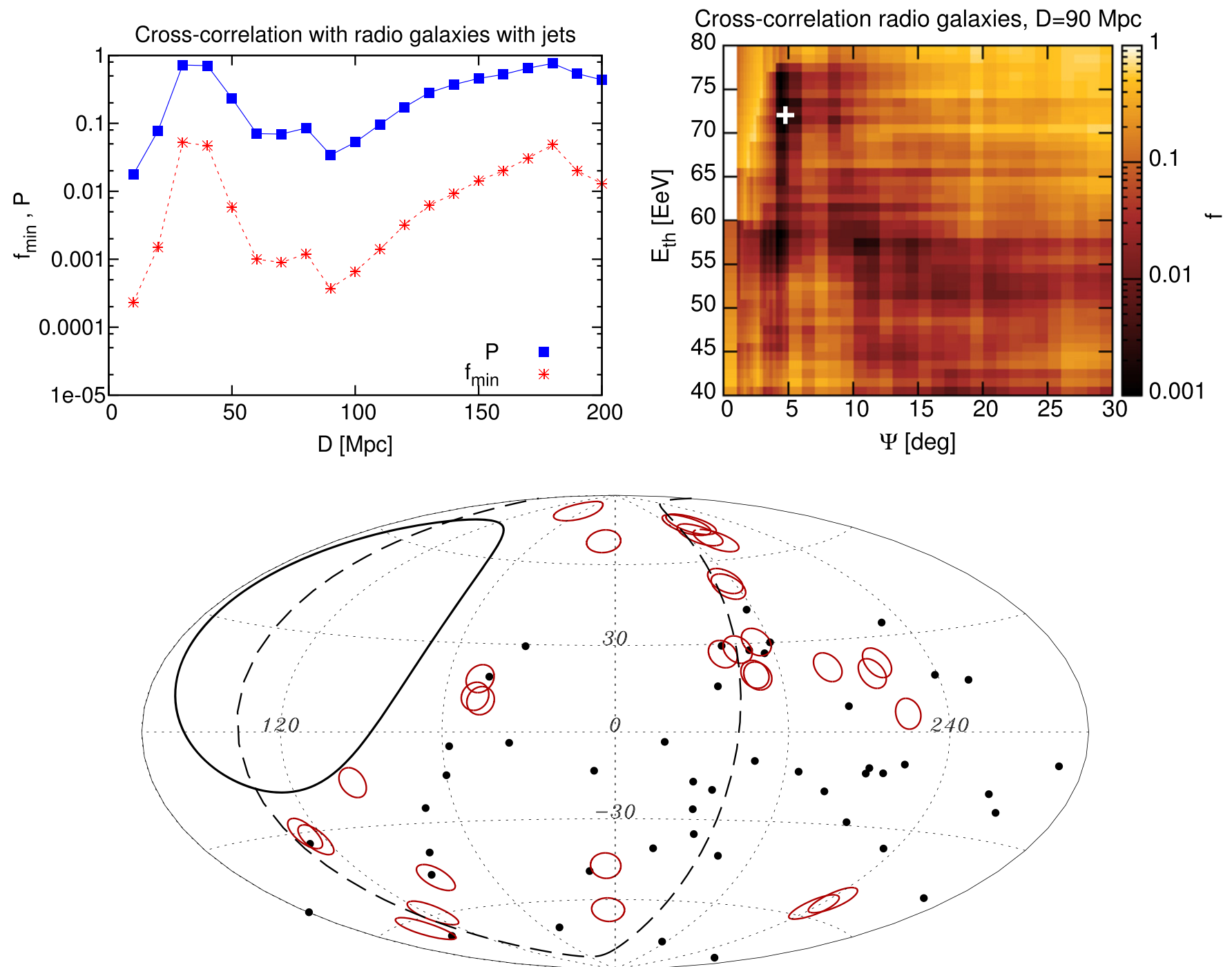

Figure 7. Cross-correlation of events with the AGNs in the catalog of radio galaxies with jets. The top-left panel shows the values of $f_{\min }$ and $P$ as a function of the maximum distance, $D$, to the AGNs considered. The top-right panel shows the results of the scan in $\psi$ and $E_{\mathrm{th}}$ for the value $D=90 \mathrm{Mpc}$ corresponding to the (second) minimum in the top-left plot. The bottom plot shows the sky distribution (in Galactic coordinates) of the events with $E \geqslant 72 \mathrm{EeV}$ (black dots). Red circles of $4: 75$ radius are drawn around the radio galaxies closer than $90 \mathrm{Mpc}$.

subsection. The minimum value of $f_{\min }=2 \times 10^{-6}$ is obtained for $D=130 \mathrm{Mpc}$ and $\mathcal{L}>10^{44} \mathrm{erg} \mathrm{s}^{-1}$. The topright panel shows the details of the scan in $\Psi$ and $E_{\mathrm{th}}$ for $D=130 \mathrm{Mpc}$ and $\mathcal{L}>10^{44} \mathrm{erg} \mathrm{s}^{-1}$. The minimum corresponds to the values $\Psi=18^{\circ}$ and $E_{\text {th }}=58 \mathrm{EeV}$. For these parameters, there are $10 \mathrm{AGNs}$ and 155 events, and 62 pairs are obtained between them, while the isotropic expectation is 32.8 . The probability of finding values $f_{\min }<2 \times 10^{-6}$ in isotropic simulations after making the same scan on $\Psi, E_{\mathrm{th}}, \mathcal{L}_{\text {min }}$, and $D$ is $\mathcal{P} \simeq 1.3 \%$.

The bottom plot in the figure is the map of events with $E \geqslant 58 \mathrm{EeV}$ (black dots) and the Swift AGN brighter than $10^{44} \mathrm{erg} \mathrm{s}^{-1}$ that are closer than $130 \mathrm{Mpc}$, represented in the map with red circles of $18^{\circ}$ radius, which is the value of $\Psi$ found at the minimum. We see that the events that contribute most to the excess of pairs observed are those arriving from directions contained inside the circles centered on IC $4329 \mathrm{~A}$ (at $\left.(\ell, b)=\left(317: 6,30^{\circ} 9\right)\right)$, ESO 506-G027 (at $(\ell, b)=$ $\left.\left(299^{\circ} .6,35.5\right)\right)$, AX J1737.4-2907 (at $\left.(\ell, b)=\left(358^{\circ} .9,1.4\right)\right)$,
NGC $612\left(\right.$ at $\left.(\ell, b)=\left(261^{\circ} 8,-77^{\circ}\right)\right)$, and NGC 1142 (at $\left.(\ell, b)=\left(175^{\circ} 9,-49^{\circ} 9\right)\right) .{ }^{102}$

Figure 9 is similar, but for the sample of radio galaxies. The scan in luminosity leads to two minima with very similar probabilities, both for $D=90 \mathrm{Mpc}$ (see the top-left panel). The first one has $f_{\min }=5.1 \times 10^{-5}$ and corresponds to $\mathcal{L}>10^{39.33} \mathrm{erg} \mathrm{s}^{-1}, \Psi=4.75$, and $E_{\text {th }}=72 \mathrm{EeV}$, with the angle and energy being equal to the parameters already obtained in the previous subsection (Figure 7). The main difference is that $32 \mathrm{AGNs}$ remain within $90 \mathrm{Mpc}$ once the luminosity cut is imposed, compared to the original sample of $39 \mathrm{AGNs}$ in the flux-limited sample, so that the expected number of pairs becomes 2.4 , while 13 are actually observed. The second minimum has $f_{\min }=5.6 \times 10^{-5}$ and corresponds

\footnotetext{
102 One of the objects in the sample of 10 AGNs is the BLLac Mrk 421, a powerful gamma-ray emitter at $(\ell, b)=\left(179.9,65^{\circ}\right)$, which has been proposed as a candidate source for the hot spot observed by the Telescope Array (Fang et al. 2014). This object is in a low-exposure region near the border of the Auger field of view, and there are no events with $E>58 \mathrm{EeV}$ within $18^{\circ}$ of it.
} 
Cross-correlation with Swift AGN

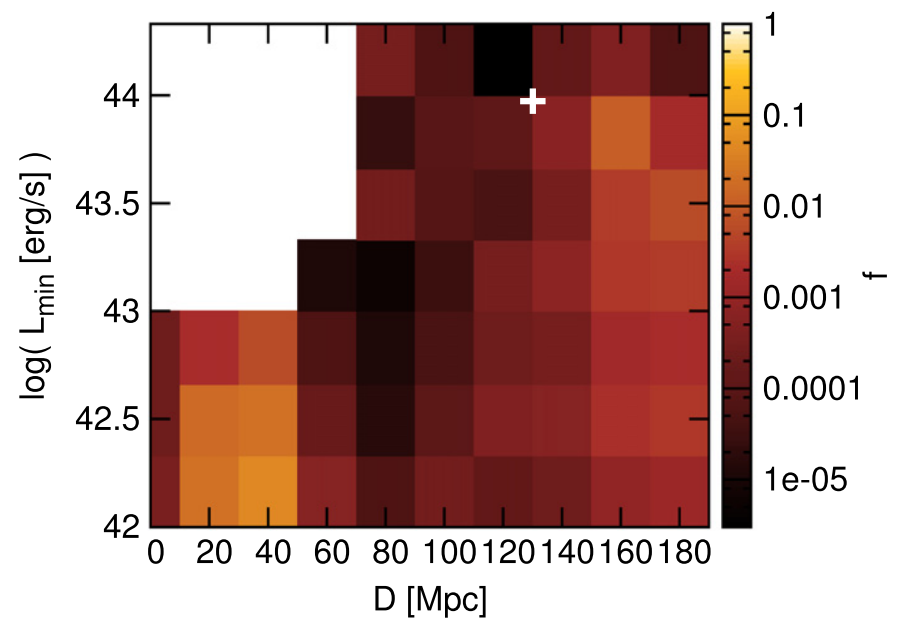

Cross-correlation Swift log $(\mathrm{L}[\mathrm{erg} / \mathrm{s}])>44, \mathrm{D}=130 \mathrm{Mpc}$

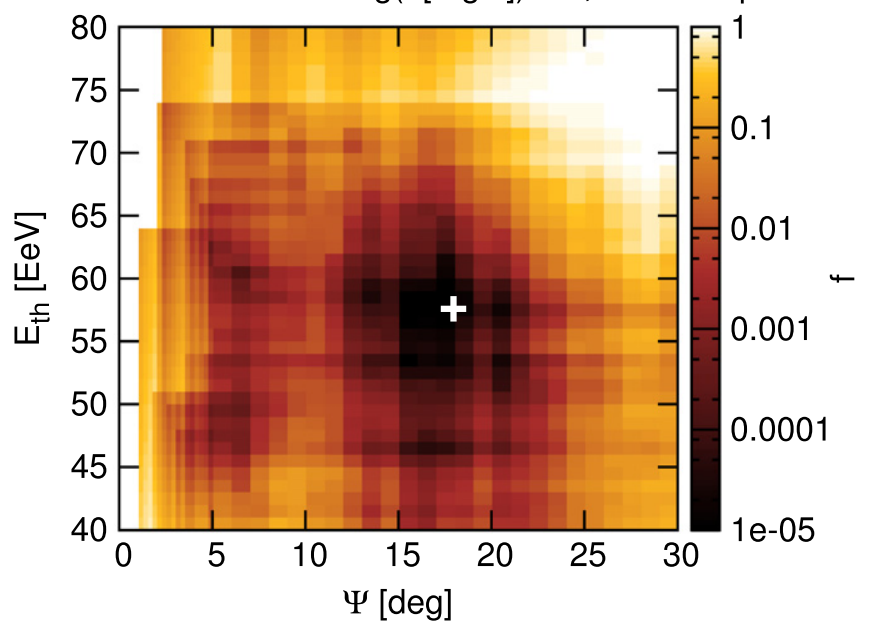

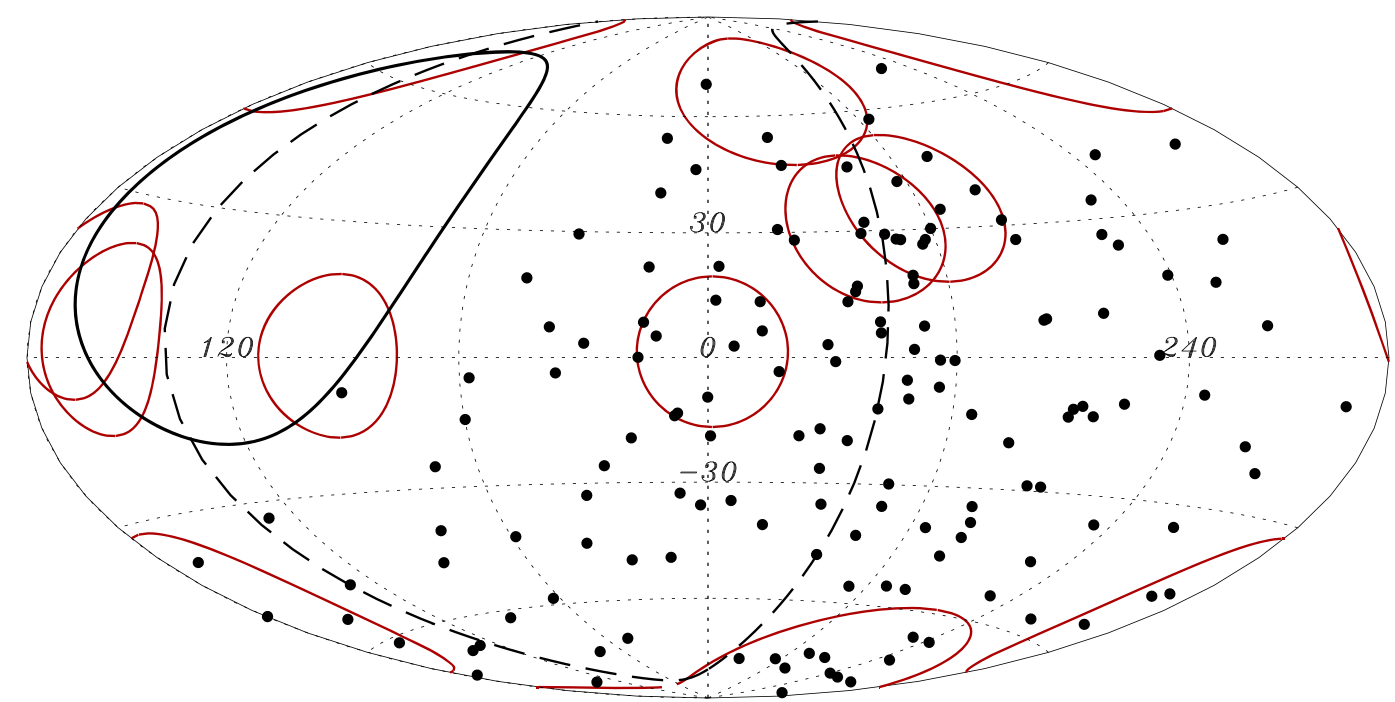

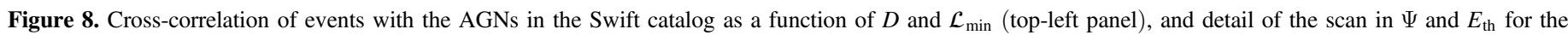

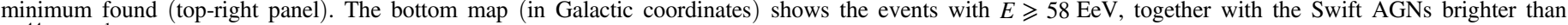
$10^{44} \mathrm{erg} \mathrm{s}^{-1}$ and closer than $130 \mathrm{Mpc}$, indicated with red circles of $18^{\circ}$ radius.

to $\mathcal{L}>10^{40} \mathrm{erg} \mathrm{s}^{-1}$. The top-right panel shows the scan in $\Psi$ and $E_{\mathrm{th}}$ for this minimum, which leads to $\Psi=12^{\circ}$ and $E_{\mathrm{th}}=58 \mathrm{EeV}$. The bottom plot shows the map of the arrival directions of the events with $E \geqslant 58 \mathrm{EeV}$ (black dots) and the radio galaxies within $90 \mathrm{Mpc}$, indicated with red circles of $12^{\circ}$ radius. We see that most of the excess in the number of pairs arises from the events falling in the circles around the radio galaxies in the Centaurus region. The globally penalized probability of getting $f_{\min }<5.1 \times 10^{-5}$ after a similar scan with the radio galaxies in this case turns out to be $\mathcal{P} \simeq 11 \%$.

\section{THE CEN A REGION}

Cen $\mathrm{A}$ is the nearest radio-loud active galaxy, at a distance of less than $4 \mathrm{Mpc}$. Thus, it is an obvious candidate source of UHECRs in the southern sky (Romero et al. 1996). In addition, the nearby Centaurus cluster is a large concentration of galaxies lying in approximately the same direction and at a distance of $\sim 50 \mathrm{Mpc}$. The most significant localized excess of UHECR arrival directions reported earlier by the Pierre Auger Collaboration (Abreu et al. 2010) was very close to the direction of Cen A. In particular, we found 13 events with energy above $55 \mathrm{EeV}$ in a circular window of radius $18^{\circ}$ centered on Cen A, while 3.2 were expected in case of isotropy. ${ }^{103}$ As shown in Section 4.1, the most significant excess observed in a blind search over the exposed sky with the present data set is also a region close to the direction of Cen $\mathrm{A}$.

In this section, we search for cross-correlations of the arrival directions with the direction of Cen $\mathrm{A},(\ell, b)=\left(-50^{\circ} .5,19^{\circ} .4\right)$. The search is performed by varying the energy threshold of events between 40 and $80 \mathrm{EeV}$ and by counting events in angular radii ranging from $1^{\circ}$ to $30^{\circ}$. To assess the significance of the observed number of events, we compare it to the one expected from isotropic simulations based on the same number of arrival directions as in the data. Figure 10 (top-left panel) shows the fraction, $f$, of those simulations that yield more than or an equal number of pairs as the data. The minimum value of $f$ is $f_{\min }=2 \times 10^{-4}$, corresponding to $E_{\text {th }}=58 \mathrm{EeV}$ and $\psi=15^{\circ}$. There are 14 events (out of a total of 155 ) observed,

\footnotetext{
103 We note, however, that the significance of the excess in this particular window of $18^{\circ}$ and for the rescaled energy threshold of $53 \mathrm{EeV}$ did not grow with the additional data included in this work, for which $n_{\mathrm{obs}} / n_{\exp }=18 / 9$, leading to a cummulative binomial probability of $4 \times 10^{-3}$.
} 

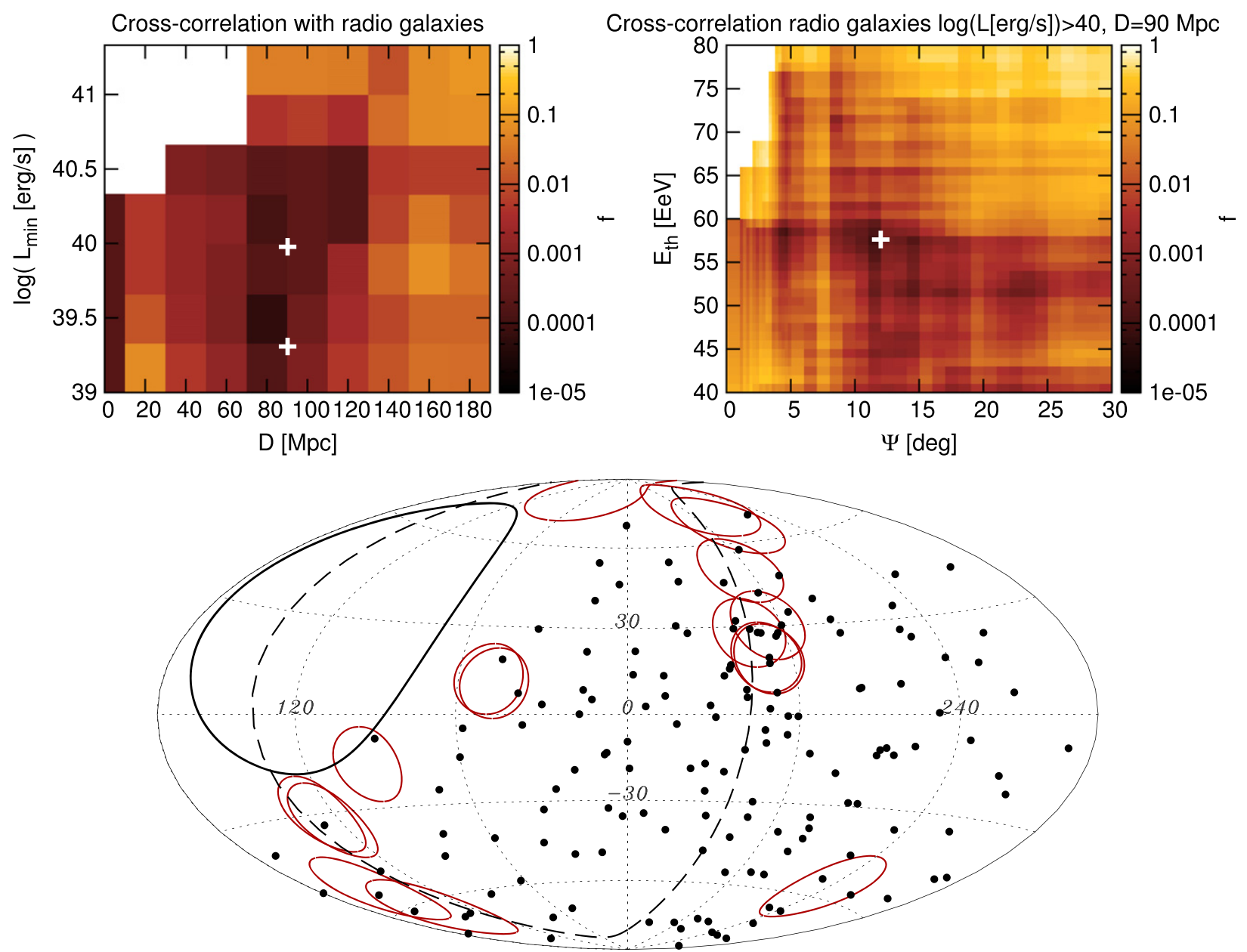

Figure 9. Cross-correlation of events with the radio galaxies as a function of $D$ and $\mathcal{L}_{\text {min }}$ (top-left panel) and detail of the scan in $\Psi$ and $E_{\text {th }}$ for the second minimum found (top-right panel). The bottom map (in Galactic coordinates) shows the events with $E \geqslant 58 \mathrm{EeV}$ together with the radio galaxies brighter than $10^{40} \mathrm{erg} \mathrm{s}^{-1}$ and closer than $90 \mathrm{Mpc}$, indicated with red circles of $12^{\circ}$ radius (i.e., the parameters of the second minimum).

while 4.5 are expected on average from isotropic distributions. The fraction of isotropic simulated data sets that yield a smaller value of $f_{\min }$ under a similar scan is $\mathcal{P} \simeq 1.4 \%$. For completeness, we show in the top-right panel of the figure the number of events with energy above $58 \mathrm{EeV}$ as a function of the angular distance from Cen A for the whole angular range, indicating also the 68,95 , and $99.7 \%$ ranges obtained with isotropic simulations. The bottom panel displays the map in Galactic coordinates of the Cen A region, showing the events with $E \geqslant 58 \mathrm{EeV}$ (black dots) and a $15^{\circ}$ radius circle around the direction of Cen A, indicated by a star.

\section{DISCUSSION}

We have presented several tests that search for signals of anisotropies in the arrival directions of the highest energy events detected by the Pierre Auger Observatory from 2004 January 1 up to 2014 March 31. The main results we obtained are summarized below.

We first updated the fraction of events with energy above $53 \mathrm{EeV}$ correlating with AGNs in the VCV catalog, obtaining a value of $28.1_{-3.6}^{+3.8} \%$, to be compared with $21 \%$ for the isotropic expectation. This test, then, does not yield significant evidence of anisotropies above this particular energy threshold. Consequently, in all other exploratory analyses we performed, we have considered the data set down to an energy of $40 \mathrm{EeV}$.
A thorough search for overdense circular regions all over the sky and for different threshold energies led to the largest deviation from isotropy in a $12^{\circ}$ radius window centered at $(\alpha, \delta)=\left(198^{\circ},-25^{\circ}\right)$ and for events with energies above $54 \mathrm{EeV}$, but more significant excesses are obtained in $69 \%$ of isotropic simulations under a similar scan. The autocorrelation of the events was also found to be compatible with the expectations from an isotropic distribution.

We found no significant excesses around the Galactic Center, the Galactic Plane, or the Super-Galactic Plane. This suggests that, if the deflections are not too large, at these energies the sources are unlikely to be Galactic and also that a non-negligible fraction of the flux arises from extragalactic sources that are not very close to the Super-Galactic Plane.

The high degree of isotropy observed in all these tests of the distribution of UHECRs is indeed quite remarkable, and certainly challenges the original expectations that assumed only few cosmic ray sources with a light composition at the highest energies. If the actual source distribution was anisotropic, these results could be understood, for instance, as due to the large deflections caused by the intervening magnetic fields if a large fraction of the CRs in this energy range were heavy, as is suggested by mass-composition studies (Abraham et al. 2010a; Aab et al. 2014b). Alternatively, it could also be explained in a scenario in which the number of individual sources 

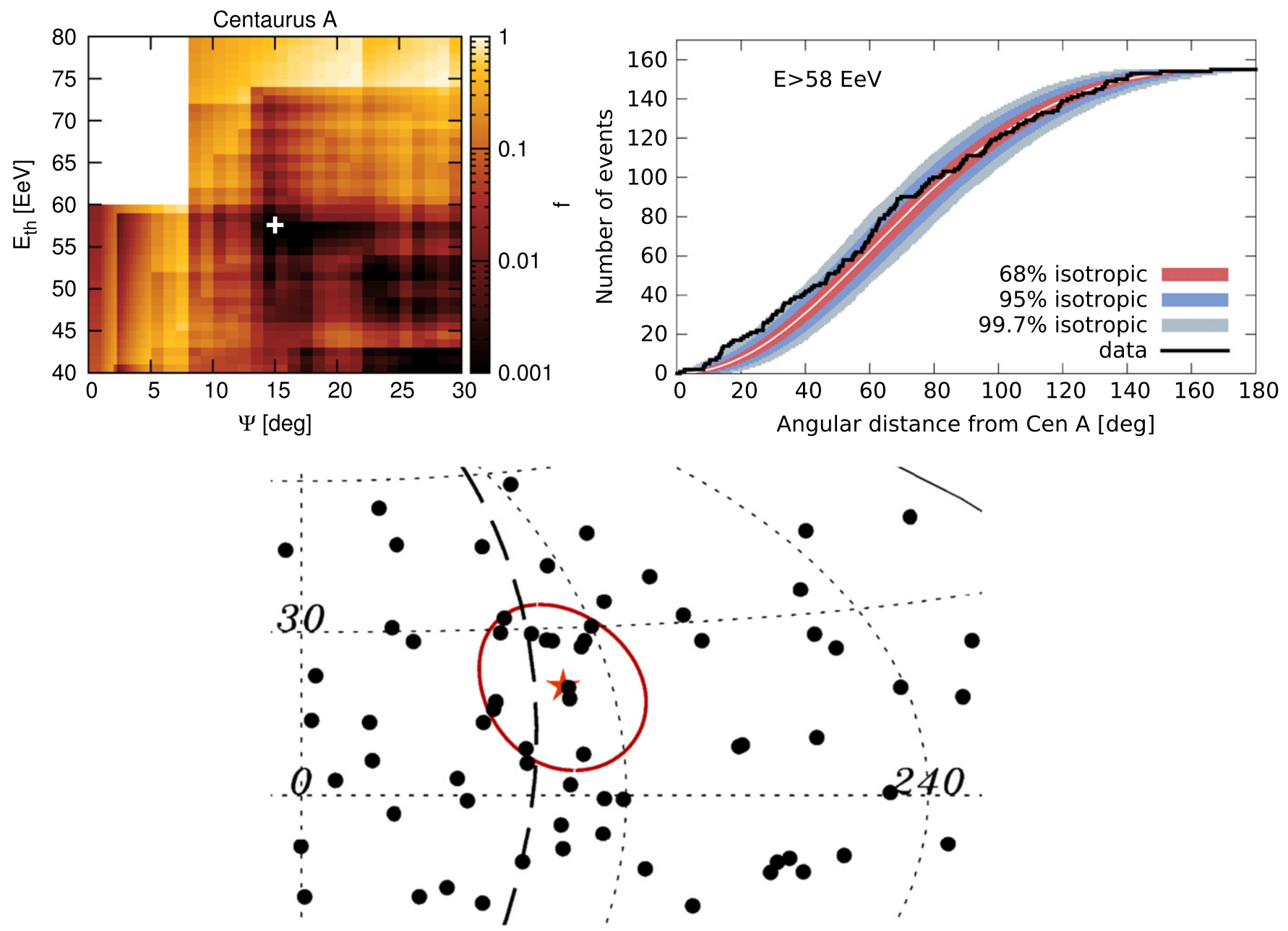

Figure 10. Correlation of events with the Cen A radio galaxy as a function of the angular distance and the energy threshold, $E_{\text {th }}$ (top-left panel). The top-right panel shows the cumulative number of events for the threshold $E_{\mathrm{th}}=58 \mathrm{EeV}$, exploring the whole angular range. The bottom panel displays the map (in Galactic coordinates) of the region around Centaurus A, showing the arrival directions of the events with $E \geqslant 58 \mathrm{EeV}$ (black dots) and a red circle of $15^{\circ}$ radius around the direction of Cen A, indicated by a star.

Table 1

Summary of the Parameters of the Minima Found in the Cross-correlation Analyses

\begin{tabular}{|c|c|c|c|c|c|c|}
\hline Objects & $\begin{array}{c}E_{\mathrm{th}} \\
(\mathrm{EeV})\end{array}$ & $\begin{array}{c}\Psi \\
\left({ }^{\circ}\right)\end{array}$ & $\begin{array}{c}D \\
(\mathrm{Mpc})\end{array}$ & $\begin{array}{c}\mathcal{L}_{\min } \\
\left(\mathrm{erg} \mathrm{s}^{-1}\right)\end{array}$ & $f_{\min }$ & $\mathcal{P}$ \\
\hline $\begin{array}{l}\text { 2MRS } \\
\quad \text { Galaxies }\end{array}$ & 52 & 9 & 90 & $\ldots$ & $1.5 \times 10^{-3}$ & $24 \%$ \\
\hline Swift AGNs & 58 & 1 & 80 & $\ldots$ & $6 \times 10^{-5}$ & $6 \%$ \\
\hline $\begin{array}{l}\text { Radio } \\
\quad \text { galaxies }\end{array}$ & 72 & 4.75 & 90 & $\ldots$ & $2 \times 10^{-4}$ & $8 \%$ \\
\hline Swift AGNs & 58 & 18 & 130 & $10^{44}$ & $2 \times 10^{-6}$ & $1.3 \%$ \\
\hline $\begin{array}{l}\text { Radio } \\
\text { galaxies }\end{array}$ & 58 & 12 & 90 & $10^{39.33}$ & $5.6 \times 10^{-5}$ & $11 \%$ \\
\hline Centaurus A & 58 & 15 & $\ldots$ & $\cdots$ & $2 \times 10^{-4}$ & $1.4 \%$ \\
\hline
\end{tabular}

contributing to the CR fluxes is large. Indeed, the lack of autocorrelation has been used in Abreu et al. (2013a) to set lower bounds on the density of sources if the deflections involved are not large.

We have also studied the cross-correlation between events and nearby extragalactic objects in different flux-limited catalogs with the aim of identifying possible scenarios of
UHECR sources. The parameters corresponding to the minima obtained when scanning in energy, distance, and angular scale are listed in Table 1 (first three rows). The penalized probabilities that these minima are due to fluctuations of an isotropic background are of the order of a few percent. In all three cases, the object distance corresponding to the minima is $D \simeq 80-90 \mathrm{Mpc}$, although it happens for different angular scales and energy thresholds. When a further scan is performed on the minimum intrinsic AGN luminosity, additional minima appear (see rows 4 and 5 in Table 1). We note that the penalized probability is $\sim 1.3 \%$ for Swift AGNs within $130 \mathrm{Mpc}$ and brighter than $10^{44} \mathrm{erg} \mathrm{s}^{-1}$, corresponding to an excess of pairs for events above $58 \mathrm{EeV}$ on angular scales of $18^{\circ}$, while for the radio galaxies the penalized probability is $\sim 11 \%$.

Finally, considering circular windows around the direction of Cen A, the most significant indication of anisotropy appears for events with $E \geqslant 58 \mathrm{EeV}$ and for an angular radius of $15^{\circ}$. After penalizing for the scan on the angle and energy threshold, this has a $1.4 \%$ probability of arising by chance from an isotropic distribution. Clearly, the events contributing to the excess around the direction of Cen $\mathrm{A}$ also contribute to the signals found in the cross-correlation searches performed 
Table A1

List of the Events with Energies Above $52 \mathrm{EeV}$ and $\theta<80^{\circ}$, the Columns Being: Year, Day, Zenith Angle $\theta$, Energy $E$, Right Ascension $\alpha$, Declination $\delta$, and Galactic Longitude $\ell$ and Latitude $b$

\begin{tabular}{|c|c|c|c|c|c|c|c|}
\hline Year & $\begin{array}{c}\text { Julian } \\
\text { days }\end{array}$ & $\begin{array}{c}\theta \\
\left({ }^{\circ}\right)\end{array}$ & $\begin{array}{c}E \\
(\mathrm{EeV})\end{array}$ & $\begin{array}{c}\alpha \\
\left({ }^{\circ}\right)\end{array}$ & $\begin{array}{c}\delta \\
\left(^{\circ}\right)\end{array}$ & $\begin{array}{c}\ell \\
\left({ }^{\circ}\right)\end{array}$ & $\begin{array}{c}b \\
\left({ }^{\circ}\right)\end{array}$ \\
\hline 2004 & 125 & 47.7 & 62.2 & 267.2 & -11.4 & 15.5 & 8.4 \\
\hline 2004 & 142 & 59.2 & 84.7 & 199.7 & -34.9 & -50.8 & 27.7 \\
\hline 2004 & 177 & 71.5 & 54.6 & 12.7 & -56.6 & -56.9 & -60.5 \\
\hline 2004 & 239 & 58.3 & 54.0 & 32.7 & -85.0 & -59.1 & -31.8 \\
\hline 2004 & 282 & 26.3 & 58.6 & 208.1 & -60.1 & -49.5 & 1.9 \\
\hline 2004 & 339 & 44.6 & 78.2 & 268.4 & -61.0 & -27.6 & -16.9 \\
\hline 2004 & 343 & 23.3 & 58.2 & 224.7 & -44.0 & -34.1 & 13.1 \\
\hline 2005 & 50 & 67.5 & 60.2 & 29.0 & -14.0 & 174.9 & -70.0 \\
\hline 2005 & 54 & 34.9 & 71.2 & 17.5 & -37.8 & -76.0 & -78.6 \\
\hline 2005 & 63 & 54.4 & 71.9 & 331.2 & -1.3 & 58.7 & -42.4 \\
\hline 2005 & 81 & 17.1 & 52.1 & 199.1 & -48.5 & -52.8 & 14.1 \\
\hline 2005 & 186 & 57.5 & 108.2 & 45.6 & -1.7 & 179.5 & -49.6 \\
\hline 2005 & 233 & 65.4 & 61.9 & 278.4 & -1.3 & 29.7 & 3.4 \\
\hline 2005 & 295 & 15.3 & 54.9 & 333.0 & -38.1 & 4.4 & -55.0 \\
\hline 2005 & 306 & 14.2 & 74.9 & 114.8 & -42.8 & -103.9 & -10.0 \\
\hline 2005 & 347 & 65.6 & 77.5 & 18.3 & 29.2 & 128.6 & -33.4 \\
\hline 2006 & 5 & 30.9 & 78.2 & 18.9 & -4.7 & 138.3 & -66.8 \\
\hline 2006 & 35 & 30.8 & 72.2 & 53.6 & -7.8 & -165.9 & -46.9 \\
\hline 2006 & 55 & 37.9 & 52.8 & 267.6 & -60.6 & -27.5 & -16.4 \\
\hline 2006 & 64 & 66.6 & 64.8 & 275.2 & -57.2 & -22.6 & -18.6 \\
\hline 2006 & 81 & 34.0 & 69.5 & 201.1 & -55.3 & -52.3 & 7.3 \\
\hline 2006 & 100 & 33.7 & 54.7 & 28.8 & -16.4 & -179.9 & -71.8 \\
\hline 2006 & 118 & 57.3 & 56.3 & 322.5 & -2.0 & 51.6 & -35.6 \\
\hline 2006 & 126 & 65.2 & 82.0 & 299.0 & 19.4 & 57.6 & -4.7 \\
\hline 2006 & 142 & 22.6 & 64.3 & 121.8 & -52.5 & -93.0 & -10.7 \\
\hline 2006 & 160 & 76.5 & 60.7 & 52.7 & -43.4 & -109.6 & -54.1 \\
\hline 2006 & 185 & 58.8 & 89.0 & 349.9 & 9.3 & 88.4 & -47.3 \\
\hline 2006 & 263 & 49.9 & 53.0 & 82.1 & 14.6 & -169.9 & -10.9 \\
\hline 2006 & 284 & 54.5 & 54.0 & 142.3 & -13.1 & -114.3 & 26.6 \\
\hline 2006 & 296 & 53.9 & 67.7 & 53.0 & -4.5 & -170.5 & -45.6 \\
\hline 2006 & 299 & 26.0 & 59.5 & 200.9 & -45.3 & -51.2 & 17.2 \\
\hline 2006 & 350 & 17.6 & 60.0 & 305.6 & -46.3 & -6.4 & -34.5 \\
\hline 2007 & 9 & 54.0 & 53.8 & 321.0 & 8.1 & 60.4 & -28.7 \\
\hline 2007 & 13 & 14.2 & 127.1 & 192.8 & -21.2 & -57.1 & 41.7 \\
\hline 2007 & 14 & 55.9 & 52.2 & 192.6 & 17.2 & -58.4 & 80.1 \\
\hline 2007 & 69 & 30.4 & 60.0 & 200.2 & -43.4 & -51.4 & 19.2 \\
\hline 2007 & 84 & 17.5 & 60.8 & 143.4 & -18.1 & -109.4 & 24.1 \\
\hline 2007 & 106 & 49.8 & 70.3 & 17.5 & 13.6 & 129.8 & -49.0 \\
\hline 2007 & 145 & 24.0 & 68.4 & 47.5 & -12.8 & -164.0 & -54.5 \\
\hline 2007 & 161 & 41.9 & 53.6 & 137.3 & 6.2 & -135.9 & 33.4 \\
\hline 2007 & 166 & 79.6 & 54.9 & 245.8 & 8.5 & 22.9 & 36.7 \\
\hline 2007 & 186 & 44.9 & 61.5 & 219.5 & -53.9 & -41.7 & 5.8 \\
\hline 2007 & 193 & 17.9 & 79.7 & 325.5 & -33.4 & 12.2 & -49.0 \\
\hline 2007 & 203 & 55.3 & 57.0 & 265.9 & 5.9 & 30.5 & 17.8 \\
\hline 2007 & 205 & 76.5 & 61.9 & 195.5 & -63.4 & -55.9 & -0.6 \\
\hline 2007 & 221 & 35.5 & 67.8 & 212.8 & -3.1 & -21.6 & 54.2 \\
\hline 2007 & 227 & 33.6 & 60.7 & 192.5 & -35.3 & -57.3 & 27.5 \\
\hline 2007 & 234 & 33.3 & 68.1 & 185.3 & -27.9 & -65.2 & 34.5 \\
\hline 2007 & 235 & 42.6 & 60.8 & 105.9 & -22.9 & -125.2 & -7.7 \\
\hline 2007 & 295 & 21.1 & 65.9 & 325.7 & -15.5 & 37.8 & -44.8 \\
\hline 2007 & 295 & 56.5 & 55.8 & 39.2 & 19.4 & 154.4 & -36.9 \\
\hline 2007 & 314 & 76.7 & 52.5 & 59.6 & 38.3 & 158.5 & -11.3 \\
\hline 2007 & 339 & 68.2 & 54.0 & 250.3 & 1.8 & 18.5 & 29.5 \\
\hline 2007 & 343 & 30.9 & 82.4 & 81.6 & -7.4 & -150.1 & -22.3 \\
\hline 2007 & 345 & 51.6 & 72.7 & 315.3 & -53.8 & -16.0 & -40.5 \\
\hline 2008 & 10 & 77.1 & 80.2 & 271.1 & 19.0 & 45.2 & 18.7 \\
\hline 2008 & 13 & 16.8 & 64.2 & 252.7 & -22.7 & -1.9 & 13.7 \\
\hline 2008 & 18 & 50.2 & 111.8 & 352.6 & -20.8 & 47.5 & -70.5 \\
\hline 2008 & 36 & 28.3 & 65.3 & 187.5 & -63.5 & -59.5 & -0.7 \\
\hline 2008 & 48 & 76.9 & 60.4 & 19.8 & -25.5 & -160.1 & -83.6 \\
\hline 2008 & 49 & 50.7 & 56.0 & 64.1 & -52.7 & -98.5 & -44.4 \\
\hline 2008 & 51 & 20.7 & 53.3 & 202.0 & -54.9 & -51.8 & 7.6 \\
\hline
\end{tabular}

Table A1

(Continued)

\begin{tabular}{|c|c|c|c|c|c|c|c|}
\hline Year & $\begin{array}{c}\text { Julian } \\
\text { days }\end{array}$ & $\begin{array}{c}\theta \\
\left(^{\circ}\right)\end{array}$ & $\begin{array}{c}E \\
(\mathrm{EeV})\end{array}$ & $\begin{array}{c}\alpha \\
\left({ }^{\circ}\right)\end{array}$ & $\begin{array}{c}\delta \\
\left({ }^{\circ}\right)\end{array}$ & $\begin{array}{c}\ell \\
\left({ }^{\circ}\right)\end{array}$ & $\begin{array}{c}b \\
\left({ }^{\circ}\right)\end{array}$ \\
\hline 2008 & 52 & 31.7 & 56.2 & 82.8 & -15.8 & -141.2 & -24 \\
\hline 2008 & 72 & 4.4 & 52.4 & 184.4 & -32.4 & -65.2 & 30 \\
\hline 2008 & 87 & 38.9 & 73.1 & 220.6 & -42.8 & -36.3 & 15 \\
\hline 2008 & 118 & 36.2 & 62.9 & 110.2 & -0.9 & -142.9 & \\
\hline 2008 & 142 & 43.4 & 56.7 & 199.4 & 6.6 & -39.0 & 68 \\
\hline 2008 & 184 & 53.7 & 55.7 & 33.0 & 11.0 & 152.8 & -47 \\
\hline 2008 & 192 & 20.2 & 55.1 & 306.5 & -55.1 & -17.1 & -35 \\
\hline 2008 & 205 & 53.1 & 56.7 & 358.9 & 15.5 & 103.6 & -45 \\
\hline 2008 & 250 & 68.8 & 52.0 & 67.7 & 4.0 & -168.7 & -28 \\
\hline 2008 & 264 & 44.4 & 89.3 & 116.0 & -50.6 & -96.4 & -12 \\
\hline 2008 & 266 & 59.0 & 61.2 & 339.4 & -63.3 & -35.4 & -47 \\
\hline 2008 & 268 & 49.8 & 118.3 & 287.7 & 1.5 & 36.5 & -3 \\
\hline 2008 & 282 & 29.0 & 58.1 & 202.2 & -16.1 & -44.2 & 45 \\
\hline 2008 & 296 & 42.8 & 64.7 & 15.6 & -17.1 & 137.9 & -79 \\
\hline 2008 & 322 & 28.4 & 62.2 & 25.0 & -61.4 & -67.1 & -54 \\
\hline 2008 & 328 & 47.2 & 63.1 & 126.4 & 5.3 & -140.8 & 23 \\
\hline 2008 & 329 & 47.9 & 66.9 & 28.9 & -2.7 & 157.9 & -61 \\
\hline 2008 & 331 & 50.7 & 52.6 & 304.4 & -26.2 & 16.7 & -29 \\
\hline 2008 & 337 & 30.8 & 65.8 & 275.2 & -14.4 & 16.7 & \\
\hline 2008 & 355 & 71.7 & 71.1 & 196.1 & -69.7 & -55.9 & -6 \\
\hline 2008 & 362 & 31.5 & 74.0 & 209.6 & -31.3 & -40.7 & 29 \\
\hline 2009 & 7 & 59.2 & 61.0 & 286.3 & -37.8 & -0.6 & -18 \\
\hline 2009 & 30 & 32.3 & 66.2 & 303.9 & -16.5 & 26.8 & -25 \\
\hline 2009 & 32 & 56.2 & 70.3 & 0.0 & -15.4 & 75.0 & -73 \\
\hline 2009 & 35 & 52.8 & 57.7 & 227.0 & -85.2 & -54.2 & -23 \\
\hline 2009 & 39 & 42.4 & 64.1 & 147.2 & -18.3 & -106.5 & 26 \\
\hline 2009 & 47 & 20.7 & 52.9 & 78.3 & -16.0 & -142.9 & -28 \\
\hline 2009 & 51 & 6.9 & 66.7 & 203.4 & -33.0 & -47.0 & 29 \\
\hline 2009 & 73 & 37.0 & 72.5 & 193.8 & -36.4 & -56.2 & 26 \\
\hline 2009 & 78 & 27.2 & 74.4 & 122.7 & -54.7 & -90.7 & -11 \\
\hline 2009 & 78 & 8.2 & 59.0 & 26.7 & -29.1 & -134.5 & -77 \\
\hline 2009 & 80 & 18.4 & 65.8 & 251.4 & -35.8 & -13.0 & \\
\hline 2009 & 80 & 44.4 & 63.8 & 170.1 & -27.4 & -80.8 & \\
\hline 2009 & 83 & 68.6 & 56.2 & 249.1 & 9.1 & 25.3 & \\
\hline 2009 & 140 & 27.2 & 55.1 & 330.8 & -8.9 & 49.5 & $-4 c$ \\
\hline 2009 & 160 & 40.9 & 52.8 & 43.9 & -25.4 & -143.4 & -6 \\
\hline 2009 & 162 & 78.2 & 70.5 & 39.4 & -34.5 & -122.6 & -66 \\
\hline 2009 & 163 & 41.2 & 71.9 & 23.3 & -40.2 & -87.9 & -74 \\
\hline 2009 & 172 & 9.7 & 65.8 & 276.1 & -33.4 & 0.1 & $-c$ \\
\hline 2009 & 191 & 26.9 & 59.5 & 294.5 & -20.5 & 19.1 & $-1 c$ \\
\hline 2009 & 197 & 51.7 & 52.2 & 129.4 & 15.2 & -149.5 & 30 \\
\hline 2009 & 202 & 60.8 & 63.6 & 358.2 & -2.8 & 90.4 & -6 \\
\hline 2009 & 212 & 52.7 & 55.3 & 122.5 & -78.5 & -68.8 & -2 \\
\hline 2009 & 219 & 40.1 & 53.2 & 29.4 & -8.6 & 166.2 & -6 \\
\hline 2009 & 219 & 59.7 & 58.3 & 304.3 & -81.9 & -48.3 & -2 \\
\hline 2009 & 237 & 78.4 & 70.0 & 325.8 & 42.8 & 90.1 & \\
\hline 2009 & 250 & 70.7 & 52.3 & 212.7 & 29.9 & 46.8 & \\
\hline 2009 & 262 & 22.4 & 58.7 & 50.1 & -25.9 & -140.5 & -56 \\
\hline 2009 & 274 & 79.4 & 82.3 & 287.7 & -64.9 & -28.9 & -26 \\
\hline 2009 & 281 & 75.5 & 75.3 & 256.7 & 14.0 & 34.2 & 2 \\
\hline 2009 & 282 & 47.2 & 60.8 & 47.6 & 11.5 & 168.6 & -38 \\
\hline 2009 & 288 & 34.2 & 58.6 & 217.9 & -51.5 & -41.6 & \\
\hline 2009 & 304 & 30.1 & 55.6 & 177.7 & -5.0 & -83.8 & \\
\hline 2009 & 335 & 64.2 & 52.5 & 171.3 & -43.8 & -73.1 & \\
\hline 2010 & 24 & 73.6 & 54.3 & 97.2 & 34.3 & 179.7 & \\
\hline 2010 & 45 & 70.0 & 61.5 & 174.7 & -21.2 & -78.9 & \\
\hline 2010 & 50 & 71.7 & 64.5 & 227.9 & -21.5 & -18.6 & \\
\hline 2010 & 52 & 52.1 & 72.9 & 258.1 & -44.9 & -17.0 & -3 \\
\hline 2010 & 72 & 43.3 & 66.9 & 278.8 & 7.9 & 38.2 & \\
\hline 2010 & 121 & 43.6 & 82.0 & 122.7 & -70.7 & -76.3 & -19 \\
\hline 2010 & 148 & 52.2 & 74.8 & 89.2 & -12.0 & -142.2 & -17 \\
\hline 2010 & 182 & 15.4 & 54.7 & 197.8 & -20.0 & -50.7 & \\
\hline 2010 & 193 & 69.6 & 58.4 & 149.2 & 5.5 & -127.5 & \\
\hline
\end{tabular}


Table A1

(Continued)

\begin{tabular}{|c|c|c|c|c|c|c|c|}
\hline Year & $\begin{array}{c}\text { Julian } \\
\text { days }\end{array}$ & $\begin{array}{c}\theta \\
\left({ }^{\circ}\right)\end{array}$ & $\begin{array}{c}E \\
(\mathrm{EeV})\end{array}$ & $\begin{array}{c}\alpha \\
\left({ }^{\circ}\right)\end{array}$ & $\begin{array}{c}\delta \\
\left({ }^{\circ}\right)\end{array}$ & $\begin{array}{c}\ell \\
\left({ }^{\circ}\right)\end{array}$ & $\begin{array}{c}b \\
\left({ }^{\circ}\right)\end{array}$ \\
\hline 2010 & 194 & 70.9 & 53.8 & 277.2 & 6.7 & 36.4 & 8.1 \\
\hline 2010 & 196 & 73.2 & 52.3 & 303.7 & -68.1 & -32.6 & -32.8 \\
\hline 2010 & 204 & 38.7 & 53.2 & 180.5 & -11.5 & -75.9 & 49 \\
\hline 2010 & 205 & 47.4 & 53.5 & 315.8 & -82.1 & -49.3 & -31.2 \\
\hline 2010 & 223 & 39.0 & 56.1 & 250.2 & -73.6 & -42.6 & -17.5 \\
\hline 2010 & 224 & 62.3 & 65.2 & 284.7 & -28.2 & 8.1 & -13.9 \\
\hline 2010 & 226 & 53.8 & 75.6 & 324.5 & 17.9 & 71.2 & -25.0 \\
\hline 2010 & 235 & 32.0 & 60.3 & 216.1 & -66.5 & -48.0 & -5.3 \\
\hline 2010 & 238 & 12.4 & 69.6 & 226.4 & -25.7 & -22.6 & 28.1 \\
\hline 2010 & 239 & 66.7 & 58.4 & 312.9 & -14.2 & 33.1 & -33.0 \\
\hline 2010 & 256 & 73.8 & 76.1 & 131.9 & -15.5 & -118.9 & 17.1 \\
\hline 2010 & 277 & 31.1 & 73.7 & 12.3 & -40.7 & -55.3 & -76.5 \\
\hline 2010 & 284 & 48.6 & 89.1 & 218.8 & -70.8 & -48.7 & -9.7 \\
\hline 2010 & 295 & 27.8 & 58.0 & 8.4 & -61.5 & -53.3 & -55.5 \\
\hline 2010 & 310 & 45.4 & 53.1 & 118.1 & 8.5 & -147.9 & 17.4 \\
\hline 2010 & 311 & 58.4 & 70.5 & 64.2 & -46.5 & -107.2 & -45.5 \\
\hline 2010 & 319 & 11.4 & 55.0 & 118.6 & -37.4 & -107.2 & -4.8 \\
\hline 2010 & 320 & 29.0 & 54.3 & 80.2 & -64.1 & -86.2 & -34.1 \\
\hline 2010 & 320 & 5.1 & 68.7 & 121.1 & -30.6 & -111.9 & 0.4 \\
\hline 2010 & 342 & 40.5 & 54.6 & 170.9 & -43.7 & -73.4 & 16.4 \\
\hline 2010 & 347 & 24.6 & 54.9 & 231.9 & -56.6 & -36.7 & 0.0 \\
\hline 2010 & 348 & 33.8 & 54.4 & 179.7 & -68.6 & -61.9 & -6.2 \\
\hline 2010 & 364 & 22.2 & 68.0 & 167.0 & -31.2 & -81.8 & 26.6 \\
\hline 2011 & 19 & 43.8 & 69.4 & 268.5 & -15.7 & 12.4 & 5.1 \\
\hline 2011 & 26 & 25.0 & 100.1 & 150.1 & -10.3 & -110.9 & 34.1 \\
\hline 2011 & 35 & 71.5 & 54.0 & 185.4 & -24.6 & -65.6 & 37.8 \\
\hline 2011 & 38 & 33.8 & 58.2 & 33.4 & -31.7 & -127.8 & -71.5 \\
\hline 2011 & 41 & 59.2 & 52.0 & 125.5 & -59.2 & -86.0 & -12.5 \\
\hline 2011 & 45 & 25.5 & 62.7 & 215.5 & -10.1 & -23.5 & 46.8 \\
\hline 2011 & 49 & 39.3 & 60.3 & 239.4 & 3.9 & 13.8 & 39.9 \\
\hline 2011 & 75 & 60.5 & 71.1 & 230.3 & 1.5 & 3.8 & 45.9 \\
\hline 2011 & 86 & 59.4 & 56.2 & 160.3 & -3.1 & -108.3 & 46.4 \\
\hline 2011 & 106 & 78.2 & 81.4 & 308.8 & 16.1 & 59.9 & -14.3 \\
\hline 2011 & 111 & 65.6 & 69.7 & 30.3 & 3.8 & 154.2 & -54.8 \\
\hline 2011 & 113 & 71.5 & 54.8 & 295.1 & -27.6 & 12.2 & -22.3 \\
\hline 2011 & 119 & 53.0 & 67.3 & 255.4 & -5.1 & 14.8 & 21.6 \\
\hline 2011 & 120 & 49.8 & 72.1 & 84.9 & 14.4 & -168.3 & -8.7 \\
\hline 2011 & 132 & 10.6 & 56.8 & 39.5 & -29.9 & -134.1 & -66.5 \\
\hline 2011 & 136 & 54.1 & 64.9 & 333.8 & -79.2 & -48.7 & -35.3 \\
\hline 2011 & 162 & 72.4 & 55.9 & 132.8 & 12.9 & -145.5 & 32.4 \\
\hline 2011 & 203 & 29.9 & 77.9 & 120.8 & -56.3 & -89.8 & -13.2 \\
\hline 2011 & 207 & 65.0 & 56.4 & 344.5 & -19.9 & 42.3 & -63.1 \\
\hline 2011 & 215 & 34.5 & 68.3 & 245.4 & -18.2 & -2.8 & 21.8 \\
\hline 2011 & 221 & 2.9 & 70.8 & 139.8 & -35.8 & -98.2 & 9.6 \\
\hline 2011 & 240 & 46.5 & 58.8 & 219.1 & -41.9 & -36.9 & 16.8 \\
\hline 2011 & 252 & 24.5 & 80.9 & 283.7 & -28.6 & 7.4 & -13.2 \\
\hline 2011 & 294 & 31.8 & 75.6 & 77.2 & -41.0 & -114.4 & -36.1 \\
\hline 2011 & 307 & 40.7 & 52.4 & 313.5 & -16.6 & 30.7 & -34 \\
\hline 2011 & 309 & 38.8 & 63.3 & 26.1 & -32.2 & -120.2 & -77.4 \\
\hline 2011 & 316 & 31.0 & 70.2 & 4.6 & -37.9 & -26.2 & -77.2 \\
\hline 2011 & 318 & 36.7 & 57.2 & 148.8 & -13.0 & -109.6 & 31.4 \\
\hline 2011 & 360 & 36.1 & 67.4 & 305.5 & -34.5 & 7.6 & -32.7 \\
\hline 2011 & 361 & 47.6 & 92.8 & 343.4 & -71.6 & -44.9 & -42.6 \\
\hline 2011 & 364 & 51.7 & 64.8 & 207.1 & -29.1 & -42.4 & 32 \\
\hline 2012 & 12 & 31.8 & 62.4 & 15.3 & -3.6 & 129.0 & -66.3 \\
\hline 2012 & 52 & 23.8 & 66.1 & 33.2 & -59.0 & -75.3 & -55.2 \\
\hline 2012 & 81 & 47.3 & 99.0 & 309.4 & -66.8 & -31.5 & -35.2 \\
\hline 2012 & 103 & 67.5 & 70.4 & 154.0 & -46.3 & -83.1 & 8.6 \\
\hline 2012 & 109 & 25.9 & 62.6 & 37.8 & -39.5 & -110.0 & -65.9 \\
\hline 2012 & 132 & 62.3 & 58.5 & 189.0 & -5.1 & -64.1 & 57 \\
\hline 2012 & 154 & 65.8 & 58.7 & 37.0 & -75.8 & -64.6 & -39.9 \\
\hline 2012 & 155 & 64.3 & 60.0 & 245.4 & -30.9 & -12.7 & 13.3 \\
\hline 2012 & 162 & 58.5 & 83.8 & 26.8 & -24.8 & -154.6 & -77.3 \\
\hline
\end{tabular}

Table A1

(Continued)

\begin{tabular}{|c|c|c|c|c|c|c|c|}
\hline Year & $\begin{array}{c}\text { Julian } \\
\text { days }\end{array}$ & $\begin{array}{c}\theta \\
\left(^{\circ}\right)\end{array}$ & $\begin{array}{c}E \\
(\mathrm{EeV})\end{array}$ & $\begin{array}{c}\alpha \\
\left(^{\circ}\right)\end{array}$ & $\begin{array}{c}\delta \\
\left(^{\circ}\right)\end{array}$ & $\begin{array}{c}\ell \\
\left(^{\circ}\right)\end{array}$ & $\begin{array}{c}b \\
\left({ }^{\circ}\right)\end{array}$ \\
\hline 2012 & 183 & 59.8 & 61.8 & 259.8 & -32.7 & -6.2 & 2.7 \\
\hline 2012 & 189 & 31.4 & 61.1 & 18.7 & -42.5 & -72.9 & -73.9 \\
\hline 2012 & 193 & 65.5 & 54.4 & 342.9 & -6.5 & 63.4 & -54.8 \\
\hline 2012 & 206 & 61.6 & 56.8 & 310.6 & -83.1 & -50.0 & -30.2 \\
\hline 2012 & 211 & 50.0 & 58.7 & 177.2 & 12.5 & -105.1 & 69.3 \\
\hline 2012 & 301 & 38.5 & 53.3 & 56.3 & -3.2 & -169.2 & -42.1 \\
\hline 2012 & 332 & 48.1 & 71.1 & 227.6 & 11.9 & 14.7 & 54.0 \\
\hline 2013 & 11 & 17.0 & 55.7 & 217.1 & -24.5 & -30.5 & 33.3 \\
\hline 2013 & 27 & 26.5 & 62.7 & 200.9 & -34.6 & -49.6 & 27.8 \\
\hline 2013 & 27 & 47.6 & 70.7 & 56.6 & -67.8 & -77.6 & -41.7 \\
\hline 2013 & 31 & 67.3 & 53.2 & 314.9 & -67.3 & -32.8 & -37.1 \\
\hline 2013 & 36 & 74.7 & 73.6 & 267.5 & -68.3 & -34.8 & -19.7 \\
\hline 2013 & 52 & 60.7 & 71.9 & 73.7 & -20.5 & -139.8 & -34.4 \\
\hline 2013 & 70 & 41.9 & 53.9 & 154.3 & -15.8 & -102.7 & 33.1 \\
\hline 2013 & 119 & 61.5 & 62.1 & 138.6 & 26.1 & -158.8 & 41.9 \\
\hline 2013 & 132 & 59.3 & 57.3 & 357.0 & -81.1 & -54.1 & -35.7 \\
\hline 2013 & 134 & 44.9 & 85.3 & 123.4 & -6.2 & -131.7 & 15.1 \\
\hline 2013 & 144 & 49.8 & 54.3 & 33.3 & -39.0 & -107.2 & -69.2 \\
\hline 2013 & 163 & 44.6 & 52.2 & 0.4 & -68.1 & -50.1 & -48.3 \\
\hline 2013 & 175 & 50.6 & 58.9 & 211.1 & 15.0 & 1.0 & 69.1 \\
\hline 2013 & 190 & 57.3 & 68.8 & 64.7 & -70.1 & -77.0 & -38.0 \\
\hline 2013 & 191 & 8.8 & 67.3 & 308.1 & -39.5 & 2.1 & -35.7 \\
\hline 2013 & 222 & 63.4 & 61.5 & 240.3 & -68.9 & -41.3 & -12.1 \\
\hline 2013 & 224 & 47.9 & 63.4 & 345.4 & -9.0 & 62.7 & -58.3 \\
\hline 2013 & 247 & 54.7 & 84.8 & 154.6 & -46.9 & -82.4 & 8.3 \\
\hline 2013 & 249 & 30.0 & 55.5 & 160.4 & -34.8 & -85.2 & 20.9 \\
\hline 2013 & 249 & 55.0 & 65.4 & 92.1 & -64.1 & -86.4 & -28.9 \\
\hline 2013 & 281 & 65.1 & 58.5 & 327.5 & -25.1 & 25.3 & -49.4 \\
\hline 2013 & 297 & 39.0 & 73.0 & 163.8 & -74.1 & -64.9 & -13.1 \\
\hline 2013 & 302 & 49.4 & 54.6 & 298.7 & 8.8 & 48.2 & -9.8 \\
\hline 2013 & 319 & 62.0 & 54.4 & 284.5 & -37.6 & -1.0 & -17.3 \\
\hline 2013 & 320 & 22.2 & 52.9 & 286.8 & -55.0 & -18.3 & -24.1 \\
\hline 2013 & 329 & 29.2 & 63.6 & 182.3 & -14.3 & -72.3 & 47.3 \\
\hline 2013 & 332 & 31.1 & 65.2 & 241.6 & -53.5 & -30.5 & -1.0 \\
\hline 2013 & 352 & 51.4 & 72.5 & 91.4 & -60.6 & -90.4 & -28.9 \\
\hline 2013 & 364 & 60.2 & 53.2 & 198.8 & -63.9 & -54.5 & -1.2 \\
\hline 2014 & 8 & 57.9 & 60.0 & 72.8 & -73.5 & -74.4 & -34.3 \\
\hline 2014 & 30 & 60.8 & 74.5 & 189.9 & -32.7 & -60.0 & 30.1 \\
\hline 2014 & 32 & 12.8 & 54.6 & 186.7 & -24.9 & -64.1 & 37.6 \\
\hline 2014 & 49 & 41.7 & 54.9 & 2.3 & -49.2 & -39.7 & -66.4 \\
\hline 2014 & 59 & 25.9 & 60.2 & 239.5 & -49.2 & -28.7 & 3.0 \\
\hline 2014 & 64 & 66.7 & 63.6 & 45.2 & -65.8 & -75.6 & -46.4 \\
\hline 2014 & 65 & 58.5 & 118.3 & 340.6 & 12.0 & 80.1 & -39.9 \\
\hline
\end{tabular}

against the different catalogs, which generally have an excess of objects in directions close to that of Cen A.

Overall, none of the tests performed yield statistically significant evidence of anisotropy in the distribution of UHECRs. In any case, it will be interesting to follow, with future data, the evolution of the excesses found in the crosscorrelation studies, particularly from Cen A and the bright AGNs.

The successful installation, commissioning, and operation of the Pierre Auger Observatory would not have been possible without the strong commitment and effort of the technical and administrative staff in Malargüe. We are very grateful to the following agencies and organizations for their financial support: Comisión Nacional de Energía Atómica, Fundación 
Antorchas, Gobierno de la Provincia de Mendoza, Municipalidad de Malargüe, NDM Holdings and Valle Las Leñas, in gratitude for their continuing cooperation over land access, Argentina; the Australian Research Council; Conselho Nacional de Desenvolvimento Científico e Tecnológico (CNPq), Financiadora de Estudos e Projetos (FINEP), Fundação de Amparo à Pesquisa do Estado de Rio de Janeiro (FAPERJ), São Paulo Research Foundation (FAPESP) Grants No. 2012/ 51015-5, 2010/07359-6, and No. 1999/05404-3, Ministério de Ciência e Tecnologia (MCT), Brazil; Grant No. MSMT-CR LG13007, No. 7AMB14AR005, No. CZ.1.05/2.1.00/03.0058, and the Czech Science Foundation Grant No. 14-17501 S, Czech Republic; Centre de Calcul IN2P3/CNRS, Centre National de la Recherche Scientifique (CNRS), Conseil Régional Ile-de-France, Département Physique Nucléaire et Corpusculaire (PNC-IN2P3/CNRS), Département Sciences de l'Univers (SDU-INSU/CNRS), Institut Lagrange de Paris (ILP) Grant No. LABEX ANR-10-LABX-63, within the Investissements d'Avenir Programme Grant No. ANR-11IDEX-0004-02, France; Bundesministerium für Bildung und Forschung (BMBF), Deutsche Forschungsgemeinschaft (DFG), Finanzministerium Baden-Württemberg, HelmholtzGemeinschaft Deutscher Forschungszentren (HGF), Ministerium für Wissenschaft und Forschung, Nordrhein Westfalen, Ministerium für Wissenschaft, Forschung und Kunst, BadenWürttemberg, Germany; Istituto Nazionale di Fisica Nucleare (INFN), Ministero dell'Istruzione, dell'Università e della Ricerca (MIUR), Gran Sasso Center for Astroparticle Physics (CFA), CETEMPS Center of Excellence, Italy; Consejo Nacional de Ciencia y Tecnología (CONACYT), Mexico; Ministerie van Onderwijs, Cultuur en Wetenschap, Nederlandse Organisatie voor Wetenschappelijk Onderzoek (NWO), Stichting voor Fundamenteel Onderzoek der Materie (FOM), Netherlands; National Centre for Research and Development, Grants No. ERA-NET-ASPERA/01/11 and No. ERA-NETASPERA/02/11, National Science Centre, Grants No. 2013/08/ M/ST9/00322, No. 2013/08/M/ST9/00728, and No. HARMONIA 5-2013/10/M/ST9/00062, Poland; Portuguese national funds and FEDER funds within Programa Operacional Factores de Competitividade through Fundação para a Ciência e a Tecnologia (COMPETE), Portugal; Romanian Authority for Scientific Research ANCS, CNDI-UEFISCDI partnership projects Grants No. 20/2012 and No. 194/2012, Grants No. 1/ASPERA2/2012 ERA-NET, No. PN-II-RU-PD-2011-30145-17, and No. PN-II-RU-PD-2011-3-0062, the Minister of National Education, Programme Space Technology and Advanced Research (STAR), Grant No. 83/2013, Romania; Slovenian Research Agency, Slovenia; Comunidad de Madrid, FEDER funds, Ministerio de Educación y Ciencia, Xunta de Galicia, European Community 7th Framework Program, Grant No. FP7-PEOPLE-2012-IEF-328826, Spain; Science and Technology Facilities Council, United Kingdom; Department of Energy, Contracts No. DE-AC02-07CH11359, No. DEFR02-04ER41300, No. DE-FG02-99ER41107, and No. DE-SC0011689, National Science Foundation, Grant No. 0450696, The Grainger Foundation, USA; NAFOSTED, Vietnam; Marie Curie-IRSES/EPLANET, European Particle Physics Latin American Network, European Union 7th Framework Program, Grant No. PIRSES-2009-GA-246806; and UNESCO.

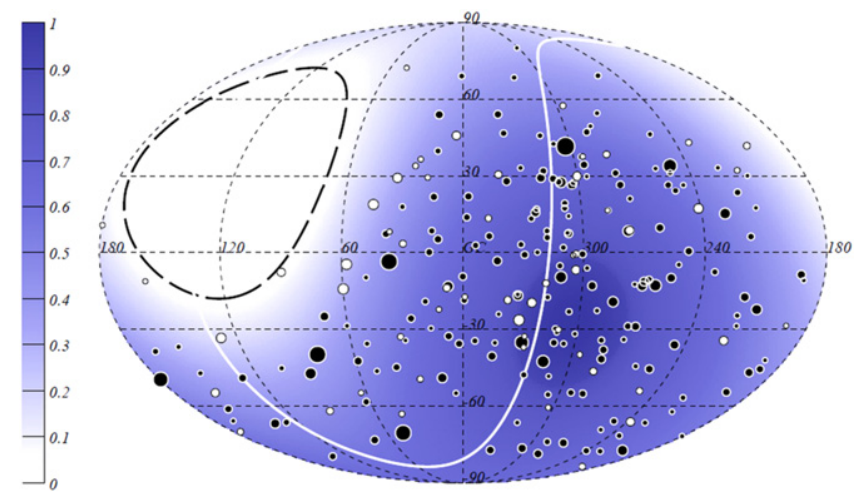

Figure A1. Map in Galactic coordinates of the arrival directions of the events with $E \geqslant 52 \mathrm{EeV}$. The black (white) circles correspond to vertical (inclined) events. The size of each circle scales with the energy of the event. The color scale is proportional to the relative exposure.

\section{APPENDIX \\ LIST OF EVENTS}

In this Appendix we give the arrival directions and energies of the 231 events $^{104}$ with $E \geqslant 52 \mathrm{EeV}$ and $\theta<80^{\circ}$ detected by the Pierre Auger Observatory from 2004 January 1 up to 2014 March 31. The threshold has been chosen so that it includes all of the events leading to the minimal probabilities in the crosscorrelation studies performed with the different catalogs. The information about these events is collected in Table A1. The different columns are: year, Julian day for that year, zenith angle, energy, right ascension, declination, Galactic longitude, and Galactic latitude.

Figure A1 displays the arrival directions of these events in Galactic coordinates. The dark filled circles correspond to the events in the vertical sample $\left(\theta \leqslant 60^{\circ}\right)$, while the white filled circles correspond to those in the inclined sample $\left(60^{\circ}<\theta<80^{\circ}\right)$. The size of the circles scales with the energy of the events. The background color in the map indicates the relative exposure of the Auger Observatory to different declinations. The white region is outside the field of view of the Auger Observatory for $\theta<80^{\circ}$.

\section{REFERENCES}

Aab, A., Abreu, P., Aglietta, M., et al. (The Pierre Auger Collaboration) 2013, arXiv:1307.5059

Aab, A., Abreu, P., Aglietta, M., et al. 2014a, JCAP, 8, 19

Aab, A., Abreu, P., Aglietta, M., et al. 2014b, PhRvD, 90, 122005

Aab, A., Abreu, P., Aglietta, M., et al. 2014, PhRvD, 90, 122006

Abbasi, R. U., Abu-Zayyad, T., Allen, M., et al. 2008, PhRvL, 100, 101101

Abbasi, R. U., Abu-Zayyad, T., Al-Seady, M., et al. 2010, PhRvL, 104, 161101

Abbasi, R. U., Abe, M., Abu-Zayyad, T., et al. 2014a, arXiv:1404.5890

Abbasi, R. U., Abe, M., Abu-Zayyad, T., et al. 2014b, arXiv:1408.1726

Abraham, J., Aglietta, M., Aguirre, I. C., et al. (The Pierre Auger Collaboration) 2004, NIMPA, 523, 50

Abraham, J., Abreu, P., Aglietta, M., et al. (The Pierre Auger Collaboration) 2007, Sci, 318, 938

Abraham, J., Abreu, P., Aglietta, M., et al. 2008a, APh, 29, 188

Abraham, J., Abreu, P., Aglietta, M., et al. 2008b, PhRvL, 101, 061101

Abraham, J., Abreu, P., Aglietta, M., et al. 2009, APh, 31, 399

Abraham, J., Abreu, P., Aglietta, M., et al. 2010a, PhRvL, 104, 091101

Abraham, J., Abreu, P., Aglietta, M., et al. 2010b, NIMPA, 613, 29

Abreu, P., Aglietta, M., Ahlers, M., et al. (The Pierre Auger Collaboration) 2013a, JCAP, 5, 9

\footnotetext{
104 We note that out of the 69 events published in Abreu et al. (2010), five turn out to have energies below $52 \mathrm{EeV}$ with the present reconstruction and, hence, they do not appear in the table.
} 
Abreu, P., Aglietta, M., , M. A., et al. (The Pierre Auger Collaboration) 2013b, JCAP, 1302, 026

Abreu, P., Aglietta, M., Ahn, E. J., et al. (The Pierre Auger Collaboration) 2010, APh, 34, 314

Abreu, P., Aglietta, M., Ahn, E. J., et al. 2011, arXiv:1107.4809

Abu-Zayyad, T., Aida, R., Allen, M., et al. 2013, ApJL, 768, L1

Ave, M., Bohacova, M., Curry, E., et al. 2013, APh, 42, 90

Ave, M. The Pierre Auger Collaboration 2007, in Proc. 30th Int. Cosmic Ray Conf. 4407 arXiv:0709.2125

Baumgartner, W. H., Tueller, J., Markwardt, C. B., et al. 2013, ApJS, 207, 19

Bonifazi, C. The Pierre Auger Collaboration 2009, NuPhS, 190, 20

Condon, J. J., Cotton, W. D., Greisen, E. W., et al. 1998, AJ, 115, 1693

Fang, K., Fujii, T., Linden, T., \& Olinto, A. V. 2014, ApJ, 794, 126

Farrar, G. R. 2014, CRPhy, 15, 339

Farrar, G. R., \& Gruzinov, A. 2009, ApJ, 693, 329
Greisen, K. 1966, PhRvL, 16, 748

Harari, D., Mollerach, S., \& Roulet, E. 2006, JCAP, 11, 12

Huchra, J. P., Macri, L. M., Masters, K. L., et al. 2012, ApJS, 199, 26

Kampert, K.-H. The Pierre Auger Collaboration 2011, in Proc. 32nd Int. Cosmic Ray Conference, arXiv:1207.4823

Li, T.-P., \& Ma, Y.-Q. 1983, ApJ, 272, 317

Mauch, T., Murphy, T., Buttery, H. J., et al. 2003, MNRAS, 342, 1117

Romero, G. E., Combi, J. A., Perez Bergliaffa, S. E., \& Anchordoqui, L. A 1996, APh, 5, 279

Tueros, M. The Pierre Auger Collaboration 2013, arXiv:1307.5059

Van Velzen, S., Falcke, H., Schellart, P., Nierstenhöfer, N., \& Kampert, K.-H. 2012, A\&A, 544, A18

Veron-Cetty, M.-P., \& Veron, P. 2006, A\&A, 455, 773

Verzi, V. The Pierre Auger Collaboration 2013, arXiv:1307.5059

Zatsepin, G., \& Kuzmin, V. 1966, JETPL, 4, 78 